\title{
Whole exome sequencing and gene expression analysis of canine osteosarcomas identify mutant TP53 and enriched immune pathways associated with longer survival
}

\section{Sunetra Das}

Colorado State University

Rupa Idate

Colorado State University

\section{Daniel Regan}

Colorado State University

Jared Fowles

Colorado State University

\section{Susan Lana}

Colorado State University

Douglas Thamm

Colorado State University

\section{Daniel Gustafson}

Colorado State University

Dawn Duval ( $\nabla$ dawn.duval@colostate.edu )

Colorado State University https://orcid.org/0000-0002-5310-0078

\section{Article}

Keywords: osteosarcoma, oncology, cancer genes

Posted Date: January 12th, 2021

DOI: https://doi.org/10.21203/rs.3.rs-135094/v1

License: (c) (1) This work is licensed under a Creative Commons Attribution 4.0 International License. Read Full License

Version of Record: A version of this preprint was published at Communications Biology on October 11th, 2021. See the published version at https://doi.org/10.1038/s42003-021-02683-0. 

1 Whole exome sequencing and gene expression analysis of canine osteosarcomas identify

2 mutant TP53 and enriched immune pathways associated with longer survival.

3

4 Authors

5 Sunetra Das ${ }^{1,2}$, Rupa Idate ${ }^{1,2}$, Daniel P. Regan ${ }^{2,4,5}$, Jared S. Fowles ${ }^{1,2,3}$, Susan E. Lana ${ }^{1,2}$, Douglas

6 H. Thamm ${ }^{1,2,5}$, Daniel L. Gustafson ${ }^{1,2,5}$, Dawn L. Duval ${ }^{1,2,5}$

8 Affiliation

$9{ }^{1}$ Department of Clinical Sciences, College of Veterinary Medicine and Biomedical Sciences, 10 Colorado State University, Fort Collins, CO, 80523, USA

$11{ }^{2}$ Flint Animal Cancer Center, Colorado State University, Fort Collins, CO, 80523, USA

$12{ }^{3}$ Cell and Molecular Biology Graduate Program, Colorado State University, Fort Collins, CO, 13 80523, USA

$14{ }^{4}$ Department of Microbiology, Immunology, \& Pathology, Colorado State University, Fort 15 Collins, CO, 80523, USA

$16{ }^{5}$ University of Colorado Cancer Center, Anschutz Medical Campus, Aurora, CO, 80045, USA

17

Corresponding author

19 Dawn Duval

20300 West Drake Road, Campus Delivery 1620, Fort Collins, CO 80523

21 970-297-4064

22 Dawn.Duval@colostate.edu

23 


\section{ABSTRACT}

Osteosarcoma affects about $2.8 \%$ of dogs with cancer, with a one-year survival rate of

27 approximately $45 \%$. The purpose of this study was to characterize the mutation and expression

28 profiles of canine osteosarcoma associated with outcome in samples from dogs treated by

29 amputation and chemotherapy.

30 The number of somatic variants identified across 26 samples ranged from 145 to 2,697 with top

31 recurrent mutations observed in TP53 (82\% of the samples) and SETD2 (22\%). Additionally, 47

32 cancer genes were identified with copy number variations in at least $58 \%$ of the samples. We

33 observed transcriptional down-regulation of myogenesis genes and up-regulation of

34 extracellular matrix genes in tumors compared to normal bone. Patients with longer disease-

35 free intervals (DFI) showed increased transcript levels of anti-tumor immune response genes.

36 Wild-type/NULL TP53 mutation status and high pre-treatment blood monocyte counts were

37 associated with a shorter DFI. Immune cell infiltration was quantified via

38 immunohistochemistry and gene expression profiling. CD3+ and MAC387+ myeloid cell

39 quantifications were not significantly associated with outcome. Expression of immune related

40 genes $P D L-1, C D 160$ and ICOS were correlated with T-cell abundance.

41 Overall, the association of gene expression and mutation profiles to outcome provides insights

42 into pathogenesis and therapeutic interventions in osteosarcoma patients.

\section{BACKGROUND}

45

Osteosarcoma (OSA) is characterized as a neoplasm of bone (mesodermal origin) and occurs

47 spontaneously in a wide spectrum of mammals including humans and dogs [1]. Most 
48 commonly, OSAs arise in the metaphysis of long bones in both dogs and humans, and

49 histologically these malignant tissues produce an extracellular matrix called tumor osteoid. This

50 malignant cancer is the most common type of bone cancer in children, adolescents, and to a

51 lesser extent in the aging population ( $>60 \mathrm{yrs}$ old), but not in adults. In comparison, $80 \%$ of

52 OSA occurs in dogs $>7$ years of age and rarely in juveniles $(6-8 \%)[2,3]$. In a study conducted in

53 Norway, the authors concluded that OSA risk was breed dependent with increased incidence in

54 large and giant breed dogs [4]. According to the Surveillance, Epidemiology, and End Results

55 (SEER; https://seer.cancer.gov/) database, the five-year survival rate in humans is 66\% but is

56 only $27 \%$ in patients with measurable metastatic disease at diagnosis. Current treatments in

57 humans include limb sparing surgery and neoadjuvant or adjuvant chemotherapy (using

58 doxorubicin, cisplatin, methotrexate, and ifosfamide) [5]. This has led to an increase in the five-

59 year survival rate from $20 \%$ to $70 \%$. Osteosarcoma in dogs is typically treated by amputation of

60 the affected limb followed by chemotherapy with doxorubicin and/or platinum-based

61 therapies. A 2014 study of 470 dogs treated for OSA with amputation and chemotherapy found

62 that the median disease-free interval (DFI) was 291 days, and was not statistically different

63 based on type of chemotherapy administered [6].

64

65 The discovery of genomic modifications that lead to malignancies and the subsequent targeting

66 of these genes or pathways is possible due to major advancements in sequencing technology

67 and resources for computational analysis. Recent articles have been published detailing the

68 genomic drivers of OSA in both humans and dogs [7-12]. The key discovery in human OSA is

69 that these cancers bear large numbers of structural and copy number variations, with little to 
no presence of activating mutations in oncogenes and infrequent point mutations in protein

71 coding genes. One of the first papers to conduct whole genome sequencing in human OSA

72 identified TP53 structural variants (SV) and single nucleotide variants (SNV) in 95\% of their

73 samples [7]. The other tumor suppressor genes with recurrent somatic variants were RB1,

74 ATRX, and DLG2 in $29 \%$ - 53\% of the patients. Perry et al., in 2014 reported similar variants;

75 however, the authors identified the PI3K/MTOR pathway as a therapeutic target, as this

76 pathway was altered in $24 \%$ of patients [8]. Further studies have used whole genome and RNA-

77 sequencing technologies in patient tumors and patient-derived xenografts to demonstrate that

78 genes with somatic copy number alterations can be targeted to reduce tumor burden [10]. To

79 date, there have been two reports of genome and exome wide variant analyses in canine OSA

$80[11,12]$. In addition to recurrent TP53 point mutations and copy number variations, these

81 studies identified two additional recurrently mutated genes: SETD2 (histone lysine

82 methyltransferase) and $D M D$ (dystrophin) that have not been previously identified in human

83 OSA. However, it is not clear if these genes represent cancer drivers in dogs. Similar to human

84 OSA, the short variant mutational burden was very low in comparison to structural (SV) and

85 copy number variants (CNV) in canine bone tumors.

86

87 Apart from surgical intervention and chemotherapy, immunotherapy is emerging as an

88 alternative form of treatment for many forms of cancer. Although, there is relatively little

89 success of immunotherapy in OSA patients to date, based on the tumor microenvironment

90 profile it is suggested that OSA might be receptive to immune-therapies [13]. Recent articles on

91 the immuno-genomic landscape in human OSA have sought to identify prognostic markers and 
92 genomic targets for immune therapy $[14,15]$. It has been shown that expression of $P D-L 1$ in

93 human OSA was significantly associated with number of immune infiltrates such as T cells,

94 dendritic cells, and natural killer cells [16]. However, the relatively low numbers of these

95 infiltrates could be one reason for low success in treating OSA patients with immunotherapy

$96[15,17]$.

97 In this paper, we have conducted multi-platform analysis of 26 canine OSA samples, including

98 whole exome sequencing, microarray analysis, and immune cell profiling. Similar to previous

99 published work, we report a prevalence of copy number variants in comparison to short

100 variants (SNVs and INDELS). The top two recurrently mutated cancer genes with short variants

101 were TP53 and SETD2. Using GISTIC2 to identify CNV, we identified a large portion of genes in

102 deleted regions of the genome and fewer amplified regions. Additionally, we identified

103 differentially expressed genes between tumors and normal metaphyseal bone based on

104 Affymetrix Canine 2.0 microarrays. The observed variant and gene expression data were

105 correlated with patient outcome data following treatment with limb amputation and

106 doxorubicin and/or platinum-based therapies. The disease-free interval (DFI) was used to

107 categorize the patients in the first (DFI $<123$ days) and fourth (DFI $>395$ days) quartiles. Tumors

108 from the first quartile group were enriched for genes in immune-related pathways. In summary,

109 the current work explores the relationship between the canine OSA mutational spectrum and

110 associated changes in gene expression to identify pathways that contribute to cancer

111 progression and therapeutic sensitivity.

112 RESULTS

113

114 Profiling the genomic landscape of canine osteosarcoma

115 
116 The whole exome sequencing data from 26 primary tumors and 26 matched normals were

117 bioinformatically analyzed to identify somatic short variants, i.e., single nucleotide variants

118 (SNVs) and insertion and deletions (INDELS), and copy number variations (CNVs). In addition to

11926 matched normal samples, 17 additional normal samples were sequenced to generate a

120 panel of normals for identification of somatic variants (Additional File 1; Table S1). To further

121 filter out germline mutations, the SNVs and INDELs reported from 722 dogs was used as a

122 germline resource during variant calling [18]. The median depth of sequencing for normal and

123 tumor samples was $247 X$ (range: $88 X-578 X$ ) and 295X (range: $126 X-453 X$ ), respectively

124 (Additional File 2, Figure S1). The total number of somatic short variants identified across 26

125 primary tumor samples ranged from 145 (T-1247) to 2,697 (T-153) (Fig. 1A). Of these variants,

$1266.9 \%$ (T-1272) to $25.9 \%$ (T-554) were annotated as protein coding variants. The protein-coding

127 mutations per megabase ranged from 0.25 (T-1272) to 7.39 (T-153) (Additional File 2, Figure

128 S2). There was no significant correlation between disease free interval (in days) and mutations

129 per megabase (Hazard Ratio: $1.095, \mathrm{p}=0.5$ ). Within the protein somatic variants identified as

130 SNVs and INDELS, an average of $80 \%$ were missense mutations (range $64 \%$ to 92\%) (Fig. 1B).

131 Overall, there were 739 deleterious and 889 tolerated missense mutations as identified by SIFT

132 scoring. We also sequenced a single metastatic tumor sample (M-1166). This sample had a total

133 of 746 somatic variants of which $10.9 \%$ were located within protein-coding regions of genes. In

134 comparison, the corresponding primary tumor (T-1166) had 908 somatic short variants and

$13514.6 \%$ of these variants were located in the gene coding regions. 
137 The CNVs were analyzed using Sequenza and GISTIC to identify significantly amplified and

138 deleted regions. The median number of genes with significant copy number alterations was

1391,468 with a range from 749 (T-1247) to 1,630 (T-153). The majority of CNVs among the 26

140 samples were gene deletions with the percentage of significantly deleted genes ranging from

$14181 \%$ (T-1247) to 90\% (T-458) of CNVs (Additional File 2, Figure S3). The number of genes with

142 CNVs in the metastatic tumor, M-1166, was 1,356, of which $91.5 \%$ were located in significantly

143 deleted regions of chromosomes. In comparison, the primary tumor, T-1166, had 1,156 CNV

144 genes with $98.6 \%$ located within the significantly deleted regions of the genome. Additionally,

145 there was no significant correlation between DFI and total CNVs for 26 samples (Hazard Ratio:

$1461.001, p=0.4)$.

147

148 The distribution of six types of single nucleotide substitutions (C.G $\rightarrow$ A.T, C.G $\rightarrow$ G.C, C.G $\rightarrow$ T.A,

$149 \mathrm{~T} \cdot \mathrm{A} \rightarrow \mathrm{A} \cdot \mathrm{T}, \mathrm{T} \cdot \mathrm{A} \rightarrow \mathrm{C} \cdot \mathrm{G}$, and $\mathrm{T} \cdot \mathrm{A} \rightarrow \mathrm{G} \cdot \mathrm{C})$ for all 26 samples revealed $\mathrm{C} \cdot \mathrm{G} \rightarrow \mathrm{T} \cdot \mathrm{A}$ transition mutations

150 as the most frequent substitution (Additional File 2, Figure S4A). There are 30 COSMIC

151 mutational signatures (v2, https://cancer.sanger.ac.uk/cosmic/signatures_v2.tt) that were

152 developed based on the distribution of the six single nucleotide substitutions within 96

153 trinucleotide contexts and pathogenesis [19]. To further delineate the mutational signatures of

154 individual samples, the non-negative matrix factorization (NMF) approach was used, and three

155 top-ranked de novo signatures (Signatures A, B, and C) were generated from the frequency of

156 those 96 types of SNVs within trinucleotide context among the 26 osteosarcoma samples (Fig.

157 1C). Signature A was most similar to COSMIC signature 9 (cosine similarity=0.72) which is

158 characterized by mutations attributed to DNA polymerase $\eta$. DNA Polymerase $\eta$ is recruited to 
159 sites lacking bases during the repair of U:G mismatches generated by activation-induced

160 cytidine deaminase through somatic hypermutation and is often found in chronic lymphocytic

161 leukemias and malignant B-cell lymphomas [20]. Signature B was similar to COSMIC signature 1

162 (cosine similarity=0.89) which is characterized by spontaneous deamination of 5-

163 methylcytosine resulting in $\mathrm{C} \rightarrow \mathrm{T}$ transitions. This signature correlates with age of cancer onset

164 in humans. Signature C was most similar to COSMIC signature 5 which is characterized by

165 transcriptional strand bias for T>C substitutions at ApTpN context and is found in most cancer

166 types. The relative contribution of trinucleotide context of de novo signatures attributed to

167 each sample indicated that $38 \%, 24 \%, 38 \%$ of samples resembled A, B, and C, respectively (Fig.

168 1D). Additionally, we have compared distribution of the trinucleotide context of 26 tumor

169 samples to the 30 known COMIC signatures. Similar to the de novo analyses, the majority of the

170 OSA samples were most similar to either signature 1 or 5; however, there was one sample with

171 highest similarity to signature 9 (T-1247). The hierarchical clustering of 30 COSMIC mutational

172 signatures suggests that the profile of tumors with signature 9 was similar to signature 5 . There

173 were four samples with highest cosine similarity for signature 6 (T-1192, T-907, T-992, T-999);

174 and one each most similar to signature 17 (T-276) and signature 19 (T-1272) (Additional File 2,

\section{Figure S4B).}

\section{Short somatic variants with SNVs and INDELs}

178 As mentioned earlier $6.9 \%-25.9 \%$ of somatic short variants were identified in protein coding

179 genes. A total of 1,579 protein coding genes had a variant in at least one of the 26 samples for a

180 total of 1,934 protein coding variants (Additional File 1, Table S2). Although there was a range 
181 of 321 (T-153) to 11 (T-1272) genes with variants across 26 samples, only 14 genes were

182 recurrently mutated in at least $15 \%$ of the samples (Additional File 2, Figure S5). There were 1831,100 genes that were mutated in only one sample and 129 genes mutated in a maximum of 184 two samples. Using DAVID (https://david.ncifcrf.gov/), we functionally characterized the genes 185 with protein coding variants to identify enriched pathways and domains (Additional File 1, 186 Table S3). The significantly enriched (Benjamini FDR <0.05) KEGG pathways included ECM187 receptor interaction (cfa04512), and Focal adhesion (cfa04510). There were two signal 188 transduction pathways enriched at p-value <0.05, PI3K-Akt signaling (cfa04151) and Calcium 189 signaling (cfa04020), in addition to cell cycle (cfa04110) and pathways in cancer (cfa05200) 190 KEGG terms. Some significantly enriched PFAM domains and Interpro classifications (Benjamini 191 FDR <0.1) were Laminin G, Spectrin repeat, Dynein heavy chain, Fibronectin type III, Laminin B, 192 and Immunoglobulin domains (see Additional File S1, Table S3 for details on genes).

194 The protein coding somatic variants were also filtered for known cancer genes using the 195 curated dataset from the COSMIC Cancer Gene Census (v91, 570 genes). On average across the 19626 samples, known cancer genes with short variants represented $5.9 \%( \pm 3.3 \%)$ of coding 197 mutations. There were 62 cancer genes with at least one variant among the 26 samples. The 198 top four genes (mutated in at least in $12 \%$ of samples) with short variant mutations were TP53, 199 SETD2 (SET domain containing 2, histone lysine methyltransferase), HSP9OAA1 (heat shock 200 protein 90kDa alpha, member A1), and DNMT3A (DNA-methyltransferase 3A) (Fig. 2A). The 201 variants identified in TP53 were primarily located within the DNA binding domain and are 202 considered to be driver mutations in human cancers (Additional file 2, Figure S6, Additional 
203 File 1, Table S4). SETD2 mutations included 3 samples with both frameshift and stop-gained 204 mutations and three samples with either a missense (variant =S1658P in T-134), frameshifts 205 (variants =P1158Lfs in T-544 and T-399; L124Yfs in T-25; Y1033Ifs inT-1087), or stop-gained 206 mutations (variant =R396* in T-544 and T-399; Q1546* in T-25; Q1431* in T-149). Homologous 207 frameshift and stop gained mutations of the SETD2 tumor suppressor gene in human cancers 208 are considered to be likely oncogenic. The missense mutation, however, identified here in 209 SETD2 is not considered to be a cancer hotspot as reported by pan-cancer analysis in cBioPortal.

210 The HSP9OAA1 gene was mutated in $12 \%$ of the samples and all three samples carried the same 211 missense mutation (A149D in T-346, T-153, T-25). Sample T-346 also carried an additional stop212 gained mutation (E530*). The only other recurrently mutated gene, DNMT3A, carried two 213 different missense mutations in three samples (N597S in T-346 and T-856; W738R in T-1023).

214 Of the 62 cancer genes, 58 genes were mutated in only one or two of the samples, limiting 215 further analysis regarding their impact on clinical outcome. By binning the tumors based upon 216 the TP53 mutation with the highest allelic frequency, we identified missense TP53 mutations in $21765 \%$ of the tumors, with frameshift or stop-gained (null) mutations identified in an additional 218 23\%, and wildtype (WT) TP53 present in 15\% of tumors (Additional File S1, Table S4). The next 219 most recurrently mutated gene, SETD2, was mutated in approximately $23 \%$ of canine OSA 220 samples. Finally, the primary tumor T-1166 had 3 different TP53 variants (D13Afs*4 at $4.5 \%$ 221 allelic frequency, L184P at 16.67\%, G234E at 7.5\%) while the subsequent metastatic lesion, M2221166 had only the L184P variant (68.2\%). Coding sequence variants in the cancer genes RANBP2 223 (RAN binding protein 2), DGCR8 (DiGeorge syndrome critical region gene 8), and FAM135B 224 (family with sequence similarity 135 member B) were also lost in the metastatic lesion, while 
225 variants in DEK (DEK proto-oncogene), FBXO11 (F-box protein 11), NOTCH2 (neurogenic locus

226 notch homolog protein 2), XPC (xeroderma pigmentosum, complementation group C), and

227 ZNF521 (zinc finger protein 521) were gained.

228

229 Copy number variants

230 In comparison to short variants, there were significantly more genes with somatic CNVs

231 (Additional File 1; Table S5). The copy number analysis was conducted using the Sequenza tool

232 to calculate gain or loss in comparison to matched normal and significantly amplified and

233 deleted genes were identified by GISTC2.0 [21]. A total of 1,662 genes were significantly

234 altered across 26 samples. The median number of genes with significant amplifications and

235 deletions was 169 (range: 114: T-1166 to 205: T-346) and 1,295 (range: 607: T-1247 to 1460: T-

236 458), respectively (Additional File 2; Figure S3). We analyzed the functional significance of

237 these genes using Enricher KEGG pathways category (https://amp.pharm.mssm.edu/Enrichr/).

238 KEGG pathways that were enriched at a q-value of 0.1 included MAPK signaling, Autophagy,

239 PI3K-AKT, p53 signaling and FOXO signaling (Additional File 1; Table S6A).

240

241 A total of 47 cancer genes (COSMIC Cancer Gene Census, v91) were identified to have

242 significantly deleted or amplified copy number aberrations (Fig. 2B). Six cancer genes were

243 significantly amplified, including, CCND3 (cyclin D3), CUX1 (cut like homeobox 1), EXT1

244 (exostosin glycosyltransferase 1), MYC (MYC proto-oncogene), RAD21 (RAD21 cohesin complex

245 component), and TFEB (transcription factor EB). Most of the remaining 41 genes were

246 significantly deleted. FOXO1 was considered to be both significantly deleted and amplified in

247 individual samples. We also functionally annotated the significantly amplified and deleted 
248 genes separately to identify pathways that might be altered by mis-regulation of these genes.

249 The two KEGG pathways enriched for the genes that were significantly amplified were cell cycle

250 and cellular senescence (adjusted p-value $<0.05$; Additional File 1, Table S6B). The top signaling

251 KEGG pathways enriched for the significantly deleted genes were p53, FoxO, PI3K-Akt, MAPK,

252 ErbB, TNF, mTOR, Wnt, Hippo, and JAK-STAT pathways (Additional File 1, Table S6C).

253

254 Correlation of copy number variation index with gene expression

255 The functional effect of copy number variations was evaluated through correlations with gene

256 expression using Pearson correlations. We correlated the gene amplitude values obtained from

257 Gistic2.0 "all_data_by_gene.txt" output to Z-scored gene expression values obtained from the

258 Affymetrix Canine 2.0 microarray data. Of the 1,662 genes with recurrent copy number

259 alterations, 1,471 genes had gene expression values from microarray data for all 26 samples.

260 There were 256 genes with significant correlations between CNV amplitude and expression

261 (Additional File 1 Table S7). The 12 cancer genes (COSMIC v91) that had significant correlation

262 were BRCA2, BUB1B, CCND1, CCND3, FLCN, FOXO1, MAP2K4, NCOR1, PTEN, RABEP1, SDHAF2,

263 and TFEB. There was a linear relationship between copy number changes and expression levels

264 of corresponding gene transcripts, which included significantly amplified genes (CCND3 and

265 TFEB) as well as significantly deleted genes including BRCA2, BUB1B, PTEN (Additional File 2,

266 Figure S7). These data suggest that although there might be a plethora of CNV changes in the

267 genome, the functional impact as evaluated by gene expression is far more limited. 
270 Using microarray analysis, we compared the transcriptome profiles of 26 canine OSA samples

271 and eight normal bone samples. Following RMA (robust multi-array average) normalization of

272 microarray data from tumor and normal bone samples, the expression data were processed to

273 identify differentially expressed genes using limma [22]. The number of up- and down-

274 regulated probes (adjusted $p$-value $<0.05$ and $\log _{2}$ fold change $> \pm 2$ ) were 201 and 721,

275 respectively (Additional File 2; Figure S8). These probes were collapsed to retain probes with

276 maximum variance for each gene and those expression values were plotted as a heatmap in Fig.

277 3A. There were 585 differentially expressed genes (DEGs) between normal bone and OSA

278 samples.

279

280 The DEGs were analyzed using pre-ranked Gene Set Enrichment Analysis (GSEA) to identify

281 enriched up- and down-regulated pathways and GO terms in the tumor samples (Additional

282 File 1; Table S8). There were 187 pathways or terms enriched at an FDR q-value of 0.05, using

283 the following MSigDB Collections: Hallmark, Canonical pathways, GO biological process, GO

284 molecular function, oncogenic signatures, immunologic signatures, and chemical and genetic

285 perturbations. The results identified enrichment of multiple gene sets associated with

286 extracellular matrix (ECM), cell proliferation, epidermal to mesenchymal transition, glycolysis,

287 and metastasis genes (Fig. 3B-C, Additional File 1 Table S8). These genes were significantly

288 upregulated in the tumor samples when compared to normal bone (positive NES score). The

289 genes downregulated in tumors were enriched for functional pathways (negative NES score)

290 including: myogenesis, innate and humoral immune response, paracrine hedgehog signaling,

291 and drug transport (Fig. 3D, Additional File 1; Table S8). The enrichment analyses also showed 
292 upregulation in tumors of genes that are increased in response to the loss of RB1 (Oncogenic

293 signature: RB_P107_DN.V1_UP). Similar to human OSA, there is loss of RB1 in canine tumors, as

294 evidenced by down-regulation of RB1 gene expression (Affymetrix Canine 2.0 microarray),

295 although copy number loss was not observed.

296

297 Immune response pathways were enriched in osteosarcomas from dogs with a longer

298 disease-free interval

299 For identification of pathways enriched in tumors from long disease-free interval (DFI) and

300 short DFI patients, we eliminated two of the 26 samples (T-474 and T-1192) due to lack of

301 follow up and sorted the remaining samples into more highly defined DFI bins based on the $25^{\text {th }}$

302 (short DFI, $<123$ days) and $75^{\text {th }}$ (long DFI $>395$ days) percentiles of DFI across the 26 samples.

303 This placed six samples in each of these two bins. The gene expression within these two bins

304 was analyzed via GSEA to identify enriched pathways. Using the same MSigDB collections used

305 for tumor vs normal analyses, 83 pathways/terms were enriched in long DFI patients. Of these

30683,80 gene sets were enriched in long DFI patients and only three gene sets were enriched in

307 short DFI patients (Table 1 and Additional File 1; Table S9). The three gene sets that were

308 enriched in short DFI patients were 1) KRAS.50_UP.V1_UP: genes upregulated in epithelial cell

309 lines due to over-expression of KRAS (Additional File 2; Figure S9A); 2) BCAT_GDS748_UP:

310 genes upregulated in kidney fibroblast cell line as a result of constitutively active $\beta$-catenin

311 (CTNNB1); and 3) SINGH_NFE2L2_TARGETS: electrophile and drug detoxification genes that are

312 down-regulated in Nrf2 knocked-down cell lines (Additional File 2; Figure S9B). These selected

313 drug detoxification genes are upregulated in short DFI patient tumors, suggesting that these 
314 patients' tumors might be more efficient in clearing chemotherapy drugs. The Hallmark gene

315 sets upregulated in long DFI patient tumors included several immune response datasets:

316 Interferon alpha and gamma response, inflammatory response, and allograft rejection along

317 with ECM gene sets (Additional File 1; Table S9, Additional File 2; Figure S10).

Table 1. Summarization of gene sets and pathways that are enriched in short or long DFI patients. See Additional File 1, Table S9 for details on enriched gene sets.

\begin{tabular}{|l|c|c|c|}
\hline $\begin{array}{l}\text { Biological process or } \\
\text { gene sets }\end{array}$ & $\begin{array}{c}\text { Number of } \\
\text { gene sets }\end{array}$ & $\begin{array}{c}\text { Average } \\
\text { NES }\end{array}$ & \begin{tabular}{c} 
Enrichment sample set \\
\hline Immune system
\end{tabular} \\
\hline Extracellular matrix & 5 & -1.88 & Long DFI patients \\
\hline BCAT_GDS748_UP & 1 & -2.04 & Long DFI patients \\
\hline KRAS.50_UP.V1_UP & 1 & 1.79 & Short DFI patients \\
\hline SINGH_NFE2L2_TARGETS & 1 & 1.92 & Short DFI patients \\
\hline
\end{tabular}

\section{Immune cell profiling in osteosarcoma}

324 To analyze the immune infiltrate in these tumors, those samples with available tissue blocks

325 were labeled with the pan-T cell marker CD3 and the myeloid cell marker MAC387 (S100A9) via

326 immunohistochemistry (Fig. 4A-D). Whole slide images were captured and immune cell density

327 as a percentage of total tumor area was determined using ImageJ software. The percent tumor 328 area positive for CD3+ T cells ranged from $0.002 \%$ (T-25, DFI - 372 days) to $4.87 \%$ (T-29C, DFI 3291197 days as of July $23^{\text {rd }}, 2020$ ) (Fig. 4E). The percent tumor area positive for MAC387+ myeloid 330 cells ranged from 0\% (T-2013, DFI - 216 days) to 3.7\% (T-74, DFI - 406 days) (Fig. 4F). Survival 331 analysis using DFI as the time event showed no statistically significant difference when the 
332 patients were stratified by high and low levels of either CD3 or MAC387 staining (Additional

333 File 2, Figure S10).

335 We also used the gene expression data for more comprehensive immunogenomic profiling of

336 the immune infiltrates in the tumor samples, given the lack of available canine-specific

337 antibodies for immune phenotyping. This analysis was performed using three different

338 methods: a) gene expression profiling for immune cell types as previously described, b)

339 CIBERSORT tool, and c) ESTIMATE R package [23-25]. As described in the Methods section, the

340 gene profiles in Rooney et al. were used to categorize immune cell subsets using signature gene

341 sets for each cell type and immune responses (Additional File 1, Table S10). Using Pearson

342 correlations, we created a matrix to identify significant associations of immune cell scores

343 (derived from gene expression), DFI, mutations per megabase and immunostaining

344 quantification of T cells (IHC T-cells) and macrophages (IHC-Macs). Statistically significant

345 positive correlations were observed between IHC T-cells and gene expression scores for

346 cytolytic activity, co-stimulation T-cell, co-inhibition T-cell, CD8+ T-cell, MHC class I, and NK cell.

347 However, IHC-Macs did not correlate with the gene expression score for macrophages (Fig. 5A).

348 Interestingly, the macrophage score was inversely correlated with CD4+ regulatory T-cells, but

349 positively correlated with neutrophils and MHC Class I. This positive correlation with

350 neutrophils is not unexpected, as MAC387 is ubiquitously expressed by all myeloid cells in the

351 dog, including neutrophils [26]. In addition, the IHC-Macs score was positively correlated with

352 co-inhibition T-cells and co-stimulation APCs. Neither DFI nor tumor mutational burden 
353 (mutations per $\mathrm{MB}$ ) were significantly correlated with these IHC staining scores or immune cell

354 gene expression signatures (Figure 5A).

355 We also took the 85 genes used for generating immune signatures and correlated them with

356 both IHC staining for T-cell and macrophage infiltration in tumors (Table 2). There were 18

357 genes that significantly correlated with T-cell infiltration at a false discovery rate of 0.05 ,

358 including the known co-inhibitory immune checkpoint molecules PDL1 (CD274), CD160 (ligand

359 for Herpesvirus entry mediator), as well as the cytotoxic T cell co-receptor CD8A (cell surface

360 marker for cytotoxic T-lymphocytes) (Table 2A). Only one gene (TNFSF4) was negatively

361 correlated with T-cell infiltration. Five genes, including PRF1, SLAMF1, OX40, TNFRSF9 and

362 CXCR3 were positively correlated with MAC387 staining. (Table 2B). The cytolytic activity

363 (derived as geometric mean of perforin and granzyme A transcript expression) in OS tumors

364 was significantly correlated with 21 immune response genes that are markers for T-cells,

365 macrophages, MHC Class I, dendritic cells, Type I and II IFN Response, B cells, and natural killer

366 cells (Additional file 1, Table S11).

367 Table 2. Correlation of immune gene expression and immunohistochemical staining of T-cell 368 and macrophage infiltration in the tumors.

369

370

2A. Gene expression correlation with CD3+ T cells in tumors.

\begin{tabular}{|l|lr|r|r|}
\hline Gene name & Associated immune cell type & \multicolumn{2}{c|}{$\begin{array}{c}\text { Correlation } \\
\text { coefficient }\end{array}$} & $\begin{array}{l}\text { p- } \\
\text { value }\end{array}$ \\
\hline CD8A & CD8+ T cells & 0.94 & 0 & 0 \\
\hline CD160 & Co-inhibition, T cell & 0.86 & 0 & 0 \\
\hline CD274 or PDL1 & Co-inhibition, APC; Co-inhibition, T cell & 0.86 & 0 & 0 \\
\hline GZMA & Cytolytic Activity & 0.75 & 0.000 & 0.003 \\
\hline CD2 & Co-stimulation, T cell & 0.7 & 0.001 & 0.010 \\
\hline ICOS & Co-stimulation, T cell & 0.68 & 0.002 & 0.015 \\
\hline CLEC5A & Macrophages & 0.67 & 0.002 & 0.016 \\
\hline TNFRSF4 & Co-stimulation, T cell & 0.65 & 0.003 & 0.020 \\
\hline
\end{tabular}




\begin{tabular}{|l|l|r|r|r|}
\hline KLRF1 & NK cells & 0.65 & 0.003 & 0.020 \\
\hline AHR & Type II IFN Response & 0.64 & 0.003 & 0.021 \\
\hline ISG20 & Type I IFN Response & 0.63 & 0.004 & 0.022 \\
\hline HAVCR2 & Co-inhibition, T cell & 0.63 & 0.004 & 0.023 \\
\hline IRF8 & pDCs & 0.6 & 0.007 & 0.031 \\
\hline CXCR3 & pDCs & 0.59 & 0.008 & 0.035 \\
\hline TAP1 & MHC Class I & 0.58 & 0.009 & 0.036 \\
\hline CD79B & B cells & 0.58 & 0.010 & 0.039 \\
\hline KDM6B & Neutrophils & 0.56 & 0.012 & 0.046 \\
\hline TNFSF4 & Co-stimulation, APC & -0.61 & 0.006 & 0.028 \\
\hline
\end{tabular}

372 2B. Gene expression correlation with MAC387+ myeloid cells in tumors.

373

\begin{tabular}{|c|c|c|c|c|}
\hline Gene name & Associated immune cell type & $\begin{array}{l}\text { Correlation } \\
\text { coefficient }\end{array}$ & $\begin{array}{l}p \text { - } \\
\text { value }\end{array}$ & $\begin{array}{l}\text { q- } \\
\text { value }\end{array}$ \\
\hline PRF1 & Cytolytic Activity & 0.74 & 0.000 & 0.008 \\
\hline SLAMF1 & Co-stimulation, APC; Co-stimulation, $\mathrm{T}$ cell & 0.71 & 0.000 & 0.011 \\
\hline CD40 or OX40 & Co-stimulation, APC & 0.68 & 0.001 & 0.020 \\
\hline TNFRSF9 & Co-stimulation, $\mathrm{T}$ cell & 0.65 & 0.002 & 0.028 \\
\hline CXCR3 & pDCs & 0.62 & 0.003 & 0.042 \\
\hline
\end{tabular}

374

375 To further delineate the immune cell profiling with a larger dataset of genes, we used the

376 CIBERSORT deconvolution tool $[24,27]$. The results of this analysis indicate a higher prevalence

377 of both resting/uncommitted $\mathrm{MO}$ and alternatively activated $\mathrm{M} 2$-polarized macrophages in all

378 the tumor samples and a lower prevalence of M1-polarized pro-inflammatory macrophages

379 (Fig. 5B). However, there was no correlation between the abundance of these three types of

380 macrophages and clinical outcome. The immunohistochemical staining for CD3+ T cells

381 positively correlated with the CIBERSORT score for four different cell types including: CD8 T

382 cells $\left(R^{2}: 0.91\right)$, activated mast cells $\left(R^{2}: 0.98\right)$, plasma cells $\left(R^{2}: 0.77\right)$, and gamma delta T cells 
$383\left(R^{2}:\right.$ 0.73). Significant correlation of MAC387 positive cells with CIBERSORT immune scores were

384 limited to follicular helper T cells ( $\left.R^{2}: 0.67\right)$.

385

386 A third method used in this study to determine levels of immune infiltrates was the ESTIMATE

387 algorithm [25]. The ESTIMATE immune score ranged from -83.61 (T-1087) 2110.4 (T-74)

388 (Fig.6C). ESTIMATE scores significantly correlated with IHC staining for both T cells (Pearson $\mathrm{R}^{2}-$

$3890.58, p$ value -0.008 ) and macrophages (Pearson $R^{2}-0.43, p$ value -0.04$)$. However, the

390 ESTIMATE scores did not correlate with genomic parameters like mutational burden, and

391 number of deleted and amplified genes. Additionally, there was no association between

392 ESTIMATE immune score and DFI.

393

394 Association of TP53 mutation status with clinical outcome

395 We observed that missense mutations in TP53 were associated with a longer disease-free

396 interval (DFI) than either the TP53 WT or null tumors following treatment by amputation of the

397 affected limb and chemotherapy with doxorubicin and/or carboplatin (Figure 6A). The median

398 DFIs for patients with mutant and WT/NULL TP53 were 296 and 95 days, respectively [HR (95\%

$399 \mathrm{Cl})=0.21$ (0.08 to 0.58), $\mathrm{p}=0.002$ ]. Along with TP53 mutation status, we also evaluated six

400 clinical co-variates that included age at diagnosis, tumor location (humerus versus other

401 locations), sex, pre-treatment peripheral blood monocyte count, serum alkaline phosphatase

402 levels and body weight to identify their association with DFI via univariate Cox proportional

403 hazards (COXPH) regression analysis. Two additional variables used in this analysis were tumor

404 immunohistochemical staining scores for CD3+ T cells and MAC387+ myeloid cells. Significantly 
increased risk was associated with two of the nine covariates, including TP53 mutation status

and tumor location (Table 3A). Although overall survival among these 26 canine OS patients

407 was not significantly reduced by increased numbers of peripheral blood monocytes (Fig. 6B),

408 using five co-variates, TP53 mutation status, tumor location, peripheral monocyte count, and

409 alkaline phosphatase level (univariate COXPH $p<0.2$ ), the final model for forward stepwise

410 COXPH regression analysis indicated that wildtype/Null TP53 mutation status and elevated

411 peripheral blood monocyte counts were predictive of a shorter DFI (Table 3B).

413 Table 3. Univariate and stepwise cox proportional hazard modelling.

414 Table 3A. Results from univariate COXPH regression analysis using six clinical and one genomic

415 parameter. The factors with a $p$-value $<0.2$ were used for the stepwise multivariate model.

\begin{tabular}{|c|c|c|c|c|c|c|c|}
\hline Variable & Group & $\begin{array}{l}\text { Median } \\
\text { survival } \\
\text { time }\end{array}$ & $\begin{array}{l}\text { Percent } \\
\text { survival - } \\
1 \text { year }\end{array}$ & $\begin{array}{l}\text { Percent } \\
\text { survival - } \\
2 \text { years }\end{array}$ & HR & $95 \%$ CI & $\begin{array}{c}\mathbf{p} \\
\text { value }\end{array}$ \\
\hline \multirow{2}{*}{ TP53 mutation status } & Mut & 296 & 47 & 12 & \multirow{2}{*}{0.21} & \multirow{2}{*}{0.08 to 0.58} & \multirow{2}{*}{0.002} \\
\hline & WT/NULL & 95 & 14 & 0 & & & \\
\hline \multirow{2}{*}{$\begin{array}{l}\text { Tumor location - } \\
\text { Humerus }\end{array}$} & Yes & 114 & 12.5 & 0 & \multirow{2}{*}{4.05} & \multirow{2}{*}{1.51 to 10.84} & \multirow{2}{*}{0.005} \\
\hline & No & 372 & 50 & 13 & & & \\
\hline \multirow{2}{*}{$\begin{array}{l}\text { Pre-treatment } \\
\text { peripheral blood } \\
\text { monocytes }(>0 r< \\
\left.0.04 * 10^{3} \text { cells } / \mu \mathrm{l}\right)\end{array}$} & High & 216 & 20 & 0 & \multirow{2}{*}{2.12} & \multirow{2}{*}{0.87 to 8.14} & \multirow{2}{*}{0.091} \\
\hline & Low & 309 & 50 & 14 & & & \\
\hline \multirow{2}{*}{$\begin{array}{l}\text { Alkaline phosphatase } \\
\text { levels }(>\text { or }<140 \\
\text { units/liter) }\end{array}$} & High & 216 & 29 & 0 & \multirow{2}{*}{1.93} & \multirow{2}{*}{0.74 to 4.99} & \multirow{2}{*}{0.176} \\
\hline & Normal & 246 & 35 & 6 & & & \\
\hline IHC CD3 staining & NA & NA & NA & NA & 0.56 & 0.23 to 1.33 & 0.191 \\
\hline Age & NA & NA & NA & NA & 1.13 & 0.93 to 1.36 & 0.224 \\
\hline \multirow{2}{*}{ Sex } & Male & 296 & 42 & 8 & \multirow{2}{*}{0.75} & \multirow{2}{*}{0.33 to 1.72} & \multirow{2}{*}{0.504} \\
\hline & Female & 142 & 33 & 8 & & & \\
\hline Weight & NA & NA & NA & NA & 1 & 0.97 to 1.04 & 0.846 \\
\hline IHC MAC387 staining & NA & NA & NA & NA & 1.02 & 0.61 to 1.69 & 0.954 \\
\hline
\end{tabular}

417 Table 3B. Results from stepwise COXPH multivariate analysis using four parameters for subset

418 selection. The two significant co-variates reported here were associated with poor prognosis.
Factors selected in stepwise model
HR
$95 \%$ CI
$p$ value 


\begin{tabular}{|l|l|l|r|}
\hline TP53 mutation status (WT/NULL) & 6.39 & 2.12 to 19.209 & 0.0009 \\
\hline Monocyte count $\left(>\mathbf{0 . 0 4} * \mathbf{1 0}^{\mathbf{3}}\right.$ cells/ $\left.\boldsymbol{\mu l}\right)$ & 2.83 & 1.09 to 7.33 & 0.032 \\
\hline
\end{tabular}

\section{DISCUSSION}

422 The current whole exome sequencing and microarray data analyses of 26 canine OSA samples

423 combines the identification of somatic variants that could drive cancer with an analysis of genes

424 with altered expression in those tumors compared to normal bone. Additionally, the clinical

425 outcome data was used to identify the pathways and somatic variants that were modulated

426 based on disease-free intervals (DFI). The mutational burden in OSA for short somatic variants

427 is usually low in both canine and pediatric cancers. The protein coding mutations per $\mathrm{Mb}$ in

428 most of the 26 canine OSA samples were similar to those reported in human OSA (less than 2

429 mutations per Mb, across 42 samples) [28]. There was only one sample (T-153) with a high

430 mutational burden, greater than 10 mutations per $\mathrm{Mb}$, which could be considered

431 hypermutable as per criteria established by Gröbner et al. 2018. In this study, neither

432 mutational burden or number of CNVs were correlated with DFI, suggesting that the absolute

433 numbers of tumor mutations were not the primary predictors of disease progression or

434 response to therapy in canine OSA.

436 Similar to previous WES and WGS studies of canine OSA $[11,12]$, we identified C>T transitions

437 as the primary point mutation type in these canine tumors. Within the context of the 96

438 trinucleotide combinations, we identified 3 de novo signatures with similarity to COSMIC

439 signatures 9, 1, and 5. Sakthikumar et al. identified COSMIC signatures 1 and 17 and found a 
440 greater representation of COSMIC signature 1, associated with aging, in Rottweilers and

441 Greyhounds, while COSMIC signature 17 and a novel signature were more common in Golden

442 Retrievers. Like the Gardner et al. study, we identified high similarity to COSMIC signature 17 in

443 only one sample (Additional Figure S4B). This sample was from a female mixed breed dog that

444 fell within the DFI <100-day group, had 1.91 protein coding mutations per megabase, and is

445 TP53 wildtype. Given the rarity of this signature in our samples, this sample was binned as a

446 signature A tumor with overall similarity to COSMIC signature 9. Another tumor, sample 1247,

447 with a high contribution of signature A was also TP53 WT, but had a DFI >300 days. While only

448 identified in the WGS analysis, the Gardner et al study also identified a group of tumors with

449 the COSMIC signature 9.

450

451 Similar to human OSA variant types, the samples in this study had more copy number variants

452 than SNVs and INDELs [7]. Over 80\% of the samples had at least one TP53 short variant and/or

453 CNV which is similar to previous reports in both human and canine studies $[8,11,12]$. We

454 identified missense TP53 mutations in 70\% (18) of the canine OSAs with nonsense or frameshift

455 variants identified in 15\% (4) of tumors and the remaining 15\% (4) being TP53 wildtype. In

456 comparison, Chen et al. identified TP53 pathway mutations in each of 20 tumors examined [7].

457 This study, with only 2 somatic missense mutations, one frameshift variant, and 55\% (11/20)

458 bearing structural variants in the first intron, suggests that for human OSA, TP53 missense

459 variants are comparatively rare. One of the surprising results from the current study was the

460 correlation of increased DFI and tumors bearing missense mutations in TP53. Similar to the

461 current association with outcome, a previous study showed that there was no significant 
462 difference in DFI between tumors with TP53 mutant and wildtype variants [29], but did identify

463 a longer overall survival in dogs with wildtype TP53. However, that study reported TP53

464 variants in only $40.7 \%$ of cases, included both missense mutations and frameshift or nonsense

465 mutations in the TP53 mutant category for DFI analysis, and not all the dogs included in these

466 analyses received chemotherapy. In addition, survival times can be confounded by the use of

467 euthanasia in companion animals, making DFI a potentially more biologically relevant measure

468 of outcome. In contrast, each of the dogs included in this study received 1 to 5 doses of

469 doxorubicin and/or 1 to 5 doses of carboplatin. A targeted exome sequencing study in human

470 small-cell lung cancer identified TP53 mutations in 54\% of patients, where it was reported that

471 the patients with mutant TP53 had longer relapse free intervals as compared to patients with

472 wild type TP53 [30]. Similarly, TP53 mutant human cancers including breast, are significantly

473 more likely to achieve pathological complete responses to chemotherapy [31-35]. Examination

474 of this phenomenon has shown that tumors from mice with the murine Tp53 R172H variant

475 exhibit greater sensitivity to doxorubicin, and fail to exit the cell cycle following treatment,

476 resulting in aberrant mitosis, and cell death [36]. Gain of function TP53 mutants, including

$477 \mathrm{R} 175 \mathrm{H}, \mathrm{R} 248 \mathrm{~W}$, and $\mathrm{R} 273 \mathrm{H}$, inactivate the ATM-dependent DNA damage response leading to

478 chromosomal translocations and a defective G2/M checkpoint, and improved treatment

479 responses [37]. This suggests that a similar phenomenon may occur in TP53 mutant canine OSA.

480 The multivariate COXPH analysis with TP53 and relevant clinical factors indicated that both high

481 numbers of pre-treatment blood monocytes and wild type/null TP53 may be poor prognostic

482 markers in canine OSA treated by amputation and doxorubicin and/or carboplatin. The higher 
483 pre-treatment monocyte counts were previously reported to be associated with a shorter DFI in 484 OSA patients [38].

486 The second most frequently mutated gene with short variants was SETD2. This gene is a 487 potential tumor suppressor and is mutated in several human solid tumors [39], but not in 488 human OSA. Although the majority of the SETD2 variants resulted in frameshifts, mutation 489 status did not impact transcript expression based on Canine 2.0 Affymetrix microarray analysis 490 in this study. The significance of these alterations at the transcript and protein level is unknown 491 at this time.

492

493 As reported in the results, a majority of genes with short variants were mutated in only one of 494 the samples, suggesting that these are passenger mutations. In addition, we did not observe 495 much overlap between the less frequently mutated genes in our study and the two other 496 canine studies $[11,12]$. The functional annotation of genes with short somatic variants showed 497 enrichment of PI3K-AKT signaling and cell cycle pathways. This is similar to functional 498 annotation of somatic variants previously identified in pediatric OSA [8].

499 As mentioned earlier, copy number variations are more prevalent in both canine and human 500 OSA. Perry et al. 2014, identified 850 genes that had significant copy number alterations in 501 pediatric OSA. Upon comparing the CNV genes with those $(1,662)$ identified here, we found 77 502 genes that overlapped, including MYC, CCND3, and TFEB. However, there were only $38 \mathrm{CNV}$ 503 genes that were common between this study and another canine whole exome sequencing 
504 study [11]. This could be due to variation across canine breeds for OSA samples and/or

505 differences in the protocols used for bioinformatics analyses.

506

507 We also identified significant correlations between the transcript levels of 257 genes (13 cancer 508 genes and 244 additional genes) and altered copy number variation amplitude, showing that 509 changes in gene copy number effects the transcription of these genes. This suggests that only 510 about $15 \%$ of CNV genes might have altered expression that is biologically relevant. Despite

511 CNVs being identified in both human samples and the current study, the amplification of MYC

512 was not significantly correlated with increased expression within the Canine 2.0 microarray

513 data. Similarly, array CGH analysis has identified recurrent MYC amplifications in canine OSA

514 that were confirmed by fluorescent in situ hybridization but were not associated with

515 elevations in MYC transcript expression [40]. Like the functional annotation of small somatic 516 variants, pathway analysis of genes with CNV showed enrichment of MAPK signaling pathway,

517 Autophagy, PI3K-Akt signaling pathway and p53 signaling pathway. All of these signaling

518 pathways are known to be altered in both canine and human OSA.

520 The differential expression analysis between normal metaphyseal bone and tumor samples

521 identified dysregulated pathways in canine OSA. Although the samples used here were primary

522 tumors, several pathways that pertain to metastatic cancer were identified. One of the major

523 gene sets up-regulated in the tumors included extracellular matrix components which included

524 integrins, collagen, proteoglycan, and glycoprotein genes. ECM dysregulation leads to

525 progression of osteosarcoma and promotes metastasis [41]. When an OSA cell line was cultured 
526 in the presence of ECM, the cells exhibited doxorubicin resistance [42] and TP53 protein levels

527 were decreased, suggesting a mechanism for drug resistance.

528 We also observed downregulation of several gene sets including myogenesis, immune

529 response, and drug transport in OSA compared to normal samples. A

530 component of the myogenesis gene set, Duchenne muscular dystrophy or dystrophin (DMD),

531 was shown to have major structural rearrangements resulting in deletion in about $50 \%$ of

532 canine OSA samples [12] analyzed using whole genome sequencing. Although these

533 rearrangements could not be detected in our WES analysis, the average expression of tumor

$534 D M D$ was significantly lower than in normal metaphyseal bone samples (Student T-test $p$ value

$535=0.006)$. Loss of DMD in $m d x$ mice is associated with reduced life span and these mice are

536 prone to spontaneously develop rhabdomyosarcomas [43]. However, the effect of DMD

537 deletion in OSA is still unknown. The other major player in pediatric OSA development is

538 deletion of RB1, which occurs in $29 \%$ of patients [7]. Although we did observe copy number

539 variation within the chromosomal region of $R B 1$, significant transcript loss was not observed.

540 However, the loss of $R B 1$ in tumor samples may have led to identification of an enriched gene

541 set (RB_P107_DN.V1_UP ) which is comprised of genes that are upregulated due to loss of RB1.

542 Based on the core enriched genes identified as RB_P107_DN.V1_UP components, canine OSA

543 had an upregulation of genes that dysregulate cell migration (SLIT3, slit guidance ligand 3),

544 extracellular matrix remodeling (COL6A1, COL5A1, COL16A1, PCOLCE), cell proliferation and

545 differentiation (FSTL1, follistatin like 1) and protein folding (FKBP10, FKBP prolyl isomerase 10). 
547 The availability of outcome data associated with each canine patient allowed for the analysis

548 and identification of pathways enriched in patients in the $25^{\text {th }}$ (short) and $75^{\text {th }}$ (long) percentiles

549 of DFI. In other words, comparison between patients that took less than 123 days or more than

550395 days to develop metastasis identified pathways associated with outcome. One of the three

551 gene sets upregulated in short DFI patients included oncogenic signature gene set,

552 KRAS.50_UP.V1_UP, which includes upregulated genes due to over-expression of oncogenic

553 KRAS. Although we did not identify a mutant variant of KRAS in the tumor samples, significant

554 upregulation of transcription factors downstream of KRAS signaling like HEY1 and ETV1 was

555 observed in short DFI patients. ETV1 is known to promote metastasis in several cancer types

556 [44-46], hence elevated levels of ETV1 in short DFI patients might play a role in accelerating

557 metastasis. In gastric cancer cell lines, upregulated ETV1 induces epithelial to mesenchymal

558 transition and increased invasiveness [47]. Similarly, mouse models of KRAS-driven pancreatic

559 cancer exhibit elevated expression of Hey1 associated with tumor progression $[48,49]$.

560 Although we did not observe an increase in mesenchymal markers like VIM (vimentin) and

$561 \mathrm{CDH} 2$ ( $\mathrm{N}$-cadherin), possibly due to the small sample size $(\mathrm{N}=6)$, the EMT gene set was enriched

562 in tumor samples when compared to normal bone samples. Additionally, another gene set

563 comprised of drug detoxification genes ( $A B C$ transporters: $A B C C 1, A B C C 2$ and NRF2 pathway

564 genes: GSR, NQO1, HMOX1, GCLC, ME1) was upregulated in short DFI patients. The $A B C C$ genes

565 are associated with multi-drug resistance [50] and the NRF2 pathway genes play a role in

566 reducing oxidative stress, thus providing defense against exogenous and endogenous stressors

567 and a survival advantage for cancer cells [51]. Drugs such as AEM1 and ML385 that disrupt the 
568 activated Nrf2 pathway, have been tested in pre-clinical cancer studies [52]. Taken together, 569 the study of NRF2 pathway in aggressive OSA can provide us with new avenues of treatment.

571 Clinical trials over the past decade have dramatically increased the relevance of cancer

572 immunotherapy, demonstrating immune checkpoint inhibitors (ICI) to be an effective

573 treatment modality in multiple human tumor types [53-57]. Despite a high degree of genomic

574 instability in OS, this unfortunately has not translated to observable clinical benefit of ICI

575 therapy in these patients, underscoring an immediate need for additional pre-clinical models

576 that allow investigation of novel therapeutic combinations that could this paradigm [7, 58-60].

577 The negative results of ICl trials in OS are surprising, given the prior clinical success of the

578 macrophage-activating innate immune stimulant Liposomal-Muramyl TriPeptide-

579 PhosphatidylEthanolamine (L-MTP-PE) immunotherapy in this disease. L-MTP-PE was

580 developed to stimulate the antitumor effects of monocytes and macrophages, and has resulted

581 in longer overall survival in both canine and human osteosarcoma patients in combination with

582 chemotherapy $[61,62]$. Due to the extensive comparative similarities between canine and

583 human OS $[63,64]$; it is very likely that clinical studies in dogs with OS also hold significant

584 potential to inform novel combination approaches for human immunotherapy studies in this

585 tumor type. To this end, we sought to characterize the immune landscape of canine OS as a

586 necessary prerequisite to informed immunotherapy trial design in this species. In this study, we

587 have identified several immune response related gene sets, primarily associated with an

588 effector \cytotoxic T cell response, which are upregulated or enriched in patients with long DFI, 589 or stated another way, decreased immune response was associated with poor outcome. Similar 
590 to our findings, a cross-species (human, dog, and mouse) transcriptome comparison conducted

591 by Scott et al. 2018, revealed a common gene cluster profiles across the three species

592 modulated in osteosarcoma tumors and cell lines [65]. Similar to the findings in this study, the

593 authors identified an association between lower transcript levels of immune-related genes and

594 poor clinical outcome. Their data further suggests that there is an association between

595 increased immune cell infiltration, namely T cells, that may be indicative of more effective

596 immune surveillance associated with prolonged impedance of progression to metastasis.

597 Due to a lack of comprehensive immunophenotyping antibody reagents in dogs, multiple

598 methods were used to comprehensively assess tumor immune cell infiltration in this study,

599 including: 1) quantitative IHC analysis of intra-tumor T cell and myeloid cell densities, and 2)

600 transcriptomic profiling of previously identified immune cell subsets by two separate methods

$601[23,24]$. The IHC staining scores for CD3+ T cells significantly correlated with transcriptomic

602 scores for CD8 T-cells and cytolytic activity, but surprisingly, there was no correlation between

603 mutational burden and the composition of the immune landscape of our canine OSA tumors,

604 specifically with regard to T cell density and cytolytic activity. However, these findings are

605 consistent with those observed in a recently published study on the immune landscape of OSA

606 human patients [15], and suggest that, in contrast to other tumor types, the degree of

607 mutational burden is likely to be a poor indicator for the presence of pre-existing anti-tumor

608 immunity or immune therapy response in OSA.

609

610 Importantly, the significant positive correlation between transcriptomic CD8 T cell and cytolytic

611 activity with CD3 immunolabeling suggests that when present, $T$ cell infiltrates in canine OSA 
612 tumors are primarily of an effector CD8 cytotoxic T cell phenotype, similar to their human

613 counterparts. These data also demonstrate that while comprehensive immunophenotyping

614 reagents for prospective immunotherapy trial design and correlative studies in dogs may be

615 limited, CD3 IHC is a feasible and cost-effective surrogate for baseline anti-tumor immune

616 response assessment in dogs. Furthermore, we observed significant associations between CD3

617 T cell infiltration and co-inhibitory immune checkpoint expression, including PD-L1, suggesting

618 that when immune responses are present, similar mechanisms of T cell immune exhaustion and

619 adaptive immune resistance are at play between human and canine tumors. While our study

620 did not find any association between increased cytolytic activity score and prolonged disease-

621 free interval in dogs with OSA, this could possibly be due to low sample size, as previous studies

622 demonstrating this association in human patients were done in high-dimensional data $[23,66]$.

623 Additionally, Wu et al. 2020 reported that the ESTIMATE immune score is negatively correlated

624 with the total number of deleted genes in human OS patients [15]. However, in our data we did

625 not observe this association. This could be due to the method for identification of deleted

626 genes in our study (whole exome sequencing) when compared to the human OSA study (whole

627 genome sequencing). Nonetheless, taken together, results of our gene set enrichment analysis

628 and immunogenomic profiling suggest the presence of both distinct subsets of so-called

629 immunologically "cold" and "hot" tumors in canine OSA, and utilization of similar mechanisms

630 of adaptive immune resistance in these tumors, providing an important foundation and

631 rationale for designing novel combination immunotherapy studies in dogs as a translational

632 strategy to improve solid tumor immunotherapy.

633 


\section{CONCLUSIONS}

635 This study analyzed the genomic and transcriptomic profiles of 26 tumors from canine OSA

636 patients and associated them with outcome data. Similar to other canine OSA studies, we have

637 identified TP53 and SETD2 as the most recurrently mutated genes. We found that in dogs

638 treated with doxorubicin and/or carboplatin, wild type/NULL TP53 mutation status was

639 associated with short DFI. The transcriptome profile of normal and tumor samples identified

640 significant upregulation of extracellular matrix genes while myogenesis was downregulated in

641 tumors. Additionally, there was decreased expression of immune response genes and higher

642 expression of KRAS and NFE2L2 targets in tumors from patients with a short DFI. The immune-

643 genomic profiling of tumors showed association of immune checkpoint genes and T cell

644 infiltration, but no association with genomic criteria such as mutational burden.

646 METHODS

\section{Sample processing - Bone tumors and matched normals}

648 Tumors from previously untreated dogs with OSA were collected along with blood, peripheral

649 blood mononuclear cells (PBMC) or stroma as matched normal samples (Additional File 1;

650 Table S1A). The samples were flash frozen and kept at $-80^{\circ} \mathrm{C}$ until processed for whole exome

651 sequencing and microarray analysis. Following the manufacturer's protocol for TRIzol

652 (Invitrogen), genomic DNA was extracted from 26 primary tumors, one metastatic tumor, and

65326 matched canine blood, PBMC or stromal samples, and DNA was cleaned using DNeasy or

654 QiaAMP DNA Blood mini kits (Qiagen). RNA was extracted from the same 26 tumor samples and

6558 normal canine bone samples using TRIzol (Invitrogen) followed by RNeasy cleanup (Qiagen) 
656 for microarray analysis (Additional File 1; Table S1B). Both RNA and DNA were quantified on a

657 NanoDrop Microvolume Spectrophotometer and quality was assessed by TapeStation or

658 Bioanalyzer (Agilent).

659

660 The whole exome DNA library was created and genomic exonic regions were captured using the 661 Agilent SureSelect XT All Exon Canine V2 (part number: 931198, Santa Clara, CA) capture kit.

662 This capture encompasses 43.45 Mb of canine exonic regions. The SureSelectXT Target

663 Enrichment System for Illumina Paired-End Multiplexed Sequencing Library kit was used to

664 create the genomic DNA library. The resultant library was sequenced on an Illumina HiSEQ4000

665 sequencer generating 151 bp paired end reads.

666 RNA samples with a RIN value $>8$ were analyzed on GeneChip Canine 2.0 Genome Arrays

667 (Affymetrix) in five separate batches.

668

669 Whole exome sequence data processing

670 Mapping Illumina reads with BWA

671 The Illumina reads were processed to identify somatic single nucleotide variants (SNVs),

672 insertions and deletions (INDELs), and copy number variations (CNVs). The 150 bp reads were

673 trimmed to eliminate low-quality (phred score <20) and adapter sequences by using the

674 Trimmomatic tool (v0.36) [67]. Both the raw and trimmed/high-quality reads were assessed by

675 FastQC (v0.11.5) [68]. The reads were then mapped against the canine genome (CanFam3.1)

676 using the BWA (v0.7.15-r1140) tool [69]. The binary alignment files (BAM) were processed to

677 mark duplicates and recalibrate bases prior to variant calling as per GATK (v4.1.2.0) best-

678 practices [70]. 
679 Indel and SNV calling using Mutect2 and post-processing of somatic variants

680 The short somatic variants, SNVs and INDELs, were called from the BAM files using Mutect2. In

681 addition to using a matched normal sample for each tumor, we created a panel of normals

682 using 43 in-house samples (Additional File 1; Table S1A). We also used 90 million population

683 variants that were called from 722 dogs as the germline resource option within Mutect2 for

684 calling somatic variants [18]. The variants were filtered using filterMutectCalls and variants

685 with a PASS notation in the FILTER column were characterized as somatic variants. The variants

686 were annotated using Ensembl Variant Effect Predictor (VEP, Ensembl version 99) and the VCF

687 file was converted to MAF (Mutation annotation file) format using the perl code: vcf2maf.pl

688 (https://github.com/mskcc/vcf2maf).

689

690 The mutational signature of each sample was deduced by using the MutationalPatterns $\mathrm{R}$

691 package (v1.12.0) [71]. Using the mut_mat function the count matrix of 96 trinucleotide

692 changes was derived from sample VCF files. To estimate the factorization rank, the non-

693 negative matrix factorization (NMF) algorithm (NMF R package) was used. The de novo

694 mutational signature was derived using the extract_signature function within the

695 MutationalPatterns package. This resulted in decomposition of the mutation count matrix into

696 three top ranked signatures and estimated the relative contribution within each sample. The

697 sample signatures were compared to 30 known COSMIC signatures by calculating the cosine

698 similarity between the NMF signatures and known cancer signatures. 
700 The somatic variants were also processed to bin protein coding and cancer gene variants. The

701 gene variants with HGVS.p variable notations were selected as protein coding genes. From this

702 list, COSMIC (version 91) was used to identify variants within known cancer genes [72]. The

703 oncoplots were plotted using R package ComplexHeatmap (v2.2.0). Pathway analysis of the

704 mutated genes was conducted using DAVID Functional Annotation Bioinformatics Microarray

705 Analysis (v6.8) tool and Enrichr to functionally annotate genes against Gene Ontology and KEGG

706 databases $[73,74]$.

707

708 Copy number variant calling using Sequenza

709 The allele-specific copy number variants were assessed using the Sequenza (v2.1.9999b1) tool

710 [75]. Briefly, the sequenza-utils bam2seqz function was used along with paired BAM files

711 (tumor and normal) to extract loci with $\mathrm{A}$ (major) and $\mathrm{B}$ (minor) allele frequencies. The

712 sequenza R package used the output of bam2seqz function for GC content normalization of

713 tumor normal depth ratio, allele-specific segmentation using the copynumber R package, model

714 fitting to infer cellularity and ploidy parameters, and copy number profiles of tumors. The

715 resulting segmentation file was annotated using the default options in the GISTIC2.0 tool to

716 identify genes in the recurrently copy number altered regions of the tumor genome. A q-value

717 cut-off of 0.01 was used to generate the final list of amplification and deletion peaks. The genes

718 associated with these peaks were identified and cancer genes (COSMIC v91) were selected for

719 plotting and cross-species comparison.

720

721 Microarray data processing 


\section{Normalization and differential expression analysis using RMA and limma}

723 Following the manufacturer's standard protocol, RNA from 26 tumor and 8 normal bone

724 samples were transcribed to cDNA, labelled and hybridized onto GeneChip ${ }^{\mathrm{TM}}$ Canine Genome

725 2.0 Array (ThemoFisher Scientific, catalog number: 900727), and scanned on an Affymetrix

726 Scanner 3000. The data files (CEL) were processed in R for normalization of probes and

727 differential expression of genes. Using simpleaffy R package (v2.62.0), the CEL files for both

728 normal and tumor samples were normalized by Robust Multi-Array Average (RMA) method,

729 which generated $\log _{2}$ expression values for 43,035 probes. The differential expression analyses

730 between normal and tumor expression were carried out using limma (v3.42.2), which used the

731 Empirical Bayes method for model fitting [22]. The significantly differentially expressed genes

732 (DEGs) were selected using a q-value (false discovery rate corrected p-value) of $<0.05$ and $\log _{2}$

733 fold change of $>2$ for upregulated and $<(-2)$ for downregulated genes, respectively.

735 GSEA analysis

736 Identification of enriched gene sets and pathways were conducted using the Gene Set

737 Enrichment Analysis (GSEA, v3.0) tool [76]. The genes sets used for this analysis were part of

738 MSigDB database, which included Hallmark (50 gene sets), Canonical pathways (2199 gene

739 sets), GO biological process (7350 gene sets), GO molecular function (1645 gene sets),

740 oncogenic signatures (189 gene sets), immunologic signatures (browse 4872 gene sets), and

741 chemical and genetic perturbations (3302 gene sets) [77]. Functional annotation of

742 differentially expressed genes from tumor and normal bone comparison was done using the

743 GSEAPreranked tool within GSEA program (FDR <0.05). In addition, we ran GSEA analysis on 
744 samples $(n=6)$ in the $25^{\text {th }}(T-856, T-907, T-1087, T-458, T-276, T-1097)$ and $75^{\text {th }}(T-74, T-1166, T-$

745346, T-399, T-29C, T-1246) percentile of DFI. Transcript expression of 15,563 genes were used

746 as input for this analysis and enriched genes sets were identified in short ( $25^{\text {th }}$ percentile) and

747 long $\left(75^{\text {th }}\right.$ percentile) DFI patients (FDR $\left.<0.05\right)$. The visualization of core enriched gene

748 expression from selected significant gene sets were plotted as heatmaps using pheatmap

749 (v1.0.12) R package [78].

750

751 Immune cell characterization

752 Immunohistochemistry

753 Archived, formalin-fixed, paraffin embedded (FFPE) tissue samples were obtained from the

754 Colorado State University Flint Animal Cancer Center Tissue Archive. Available paraffin blocks

755 were routinely processed for hematoxylin and eosin (H\&E) staining, as well as

756 immunohistochemistry. The H\&E stained slides were evaluated by a board-certified pathologist

757 (DPR) to confirm diagnosis and the presence of adequate viable tumor sample for IHC analysis.

758 Immunohistochemistry was performed via routine, automated methods on the Leica Bond Max

759 autostainer (Leica Biosystems Inc.), with the following panel of previously published canine

760 cross-reactive antibodies: rabbit polyclonal anti-human CD3 (pan T lymphocyte marker; Dako,

761 clone A0452), and monoclonal mouse anti-human Myeloid/Histiocyte antigen (MAC387;

762 monocytes/macrophages; Dako, clone MAC387). Antigen retrieval was performed using Leica

763 Epitope Retrieval 2 (Tris-EDTA buffer, pH 9) for 20 min. Detection was performed with

764 PowerVision IHC detection systems (Leica Biosystems, Inc.), using a polymeric alkaline

765 phosphatase anti-mouse IgG (MAC387) or anti-rabbit IgG (CD3) and Fast Red chromogen. 
767 Whole slide brightfield images of IHC stained slides were digitally captured using an Olympus

768 IX83 microscope at 10x magnification and fixed exposure times for all samples. Quantitative

769 image analysis was performed using open-source ImageJ software (National Institutes of

770 Health). Parent images were converted to gray scale .tiff images for analysis. Tumor tissue

771 regions-of-interest (ROIs) were segmented from adjacent normal tissue by manual outlining in

772 ImageJ in blinded fashion by a board-certified veterinary pathologist. Following determination

773 of the ROI, positively labeled infiltrating immune cells were counted using the color

774 deconvolution algorithm. Briefly, a positive pixel threshold for all immune cell markers was

775 determined visually by a veterinary pathologist using appropriate isotype-stained control slides.

776 Images were subjected to color deconvolution, followed by global, automated application of

777 this intensity threshold to all images. Following automated image analysis, positive pixel masks

778 of each image were blindly evaluated by a pathologist to ensure thresholding accuracy. Data

779 was analyzed and the number of infiltrating immune cells was expressed as a percentage of

780 total tumor tissue area.

781

782 Gene expression profiling of immune cells

783 In the last few years, multiple groups have characterized immune cell types using gene

784 expression profile. Using the gene sets for immune cell types provided by Rooney et al 2015, we

785 have calculated a score for immune cell types in each sample [23]. A score was calculated as

786 geometric mean of gene expression for signature genes of each cell type (Additional File 1,

787 Table S10). These scores were used to assess associations with DFI, mutations per megabase 
788 (tumor burden), and immune staining quantification data for T-cells and myeloid cells using

789 Pearson correlation. The significance of multiple correlations was corrected for false discovery

790 rates using Benjamini-Hochberg method (R package fdrtool, v1.2.15). The immune cell profiling

791 for tumor microenvironment was also carried out by using CIBERSORT tool [24]. Using

792 transcript expression data as input, deconvolution of 22 functionally defined immune cell types

793 was carried out [27]. A third method to calculate immune cell infiltration was done by using the

794 ESTIMATE (v1.0.13) R package for each tumor samples [25].

795

796 Survival analyses and graphical plots

797 The association of TP53 mutation status, monocyte counts, and IHC staining scores with patient

798 DFI was assessed using Kaplan-Meier survival analyses (R packages used: survival, v.3.2-3 and

799 survminer, v.0.4.8). The univariate Cox proportional hazard regression (R package: survival,

800 v.3.2-3) was used to identify significant association of clinical parameters and TP53 mutation

801 status to patient outcome. A p value cut-off of 0.2 was used to select factors for running the

802 stepwise algorithm. The stepwise forward multivariate COXPH regression was carried out by $\mathrm{R}$

803 package My.stepwise (v0.1.0). The significance level for model entry and exit was 0.1. The final

804 model was comprised of factors what were significantly associated with outcome at a p-value

805 less than 0.05 .

806 Data processing and availability

807 The whole exome data was processed on the RMACC Summit supercomputer [79]. The Illumina 808 raw fastq files were submitted to NCBI Short Read Archive (SRA) database. The canine 809 osteosarcoma and normal samples have been submitted to Bioproject PRJNA613479 and 
811 Omnibus (GEO) database using accession number GSE76127. The tools, databases, and their

812 versions, in addition to the bioinformatic pipeline used in this study can be accessed here:

813 https:/github.com/sdas2019/Canine-Osteosarcoma-Whole-Exome-Sequencing-Pipeline.

$814 \quad$ Figure legends.

815

Fig. 1. Mutational signature profile in canine osteosarcoma. A. Distribution of total short variants (SNVs and INDELs) across 26 samples. The samples were sorted in ascending order of disease-free interval (DFI) and binned in three discrete groups. B. Distribution of mutation types as identified by VEP across 26 samples. Missense mutations were the most common type of short variant in this dataset. C. Mutation signature analyses showing the relative distribution of six single nucleotide changes in 96 different trinucleotide contexts across three de novo signatures. D. Relative contribution of three predominant signatures across 26 samples.

Fig. 2. Oncoplot of cancer census genes with variants in canine osteosarcoma samples. A. The genes with short variants (SNVs and INDELS) are plotted along with sample characteristics like disease free interval (DFI) and tumor location. The cancer genes were categorized as oncogenes and tumor suppressor genes in accordance to the Cancer Gene Census. B. The genes with recurrent copy number variations (amplifications and deletions) were plotted. The samples were sorted in ascending order of DFI in both plots. Gene names colored red indicate significant correlation of copy number amplitude and transcript expression.

Fig.3. Graphical representation of differentially expressed genes (DEG). A. Heatmap of DEGs between normal bone ( $\mathrm{N}-$ ) and canine osteosarcoma samples (T-). B-D. The heatmap of coreenriched genes associated with three significantly enriched Hallmark gene sets in tumor samples in comparison to normal bone, as identified by GSEA analysis.

Fig.4. Association of TP53 mutation status and clinical parameters with outcome. A. Kaplan Meier plots for disease free interval (DFI) in dogs with osteosarcoma stratified by TP53 mutation status. The dogs with wild type/null TP53 status had a shorter DFI compared to dogs with TP53 missense mutations. B. Kaplan Meier plots for dogs with osteosarcoma stratified by pre-treatment monocyte count ( $>$ or $<0.04 * 10^{3}$ cells $/ \mu$ l). Higher monocyte counts were associated with short DFI patients. 
843 Fig 5. Representative figures of immunohistochemical staining of canine osteosarcoma samples.

844 A-B. These samples were stained for CD3+ T-cell infiltration in A (low levels - T-1023) and B

845 (high levels - T-1162). C-D. These samples were stained with the MAC387 antibody against

846 S100A9 to distinguish macrophage infiltration. C represents low levels (T-1162) and D

847 represents high levels (T-856). E-F. Quantification of immunohistochemical labelling of T-cell

848 (CD3+ cells) and Macrophages (MAC387 cells) across 19 and 22 samples, respectively.

Fig.6. Profiling of immune cell infiltration using gene expression data. A. Pearson correlation matrix of immune cell expression score and immune staining levels of T-cell (IHC percent CD3+ cells) and macrophages (IHC percent MAC387 cells). We also correlated mutations per megabases (MB) and disease-free interval (DFI) with other variables. Only the significant (FDR $<0.05)$ correlation values are plotted. B. Quantification of 22 immune cell types using CIBERSORT. The samples are sorted by DFI. The most prevalent cell type in the tumor microenvironment were $\mathrm{M} 0$ and $\mathrm{M} 2$ macrophages. $\mathrm{C}$. Immune score quantification using ESTIMATE R package.

\section{References}

1. Withrow SJ, Khanna $\mathrm{C}$ : Bridging the gap between experimental animals and humans in osteosarcoma. Cancer Treat Res 2009, 152:439-446.

2. Makielski KM, Mills LJ, Sarver AL, Henson MS, Spector LG, Naik S, Modiano JF: Risk Factors for Development of Canine and Human Osteosarcoma: A Comparative Review. Vet Sci 2019, 6.

3. Gustafson DL, Duval DL, Regan DP, Thamm DH: Canine sarcomas as a surrogate for the human disease. Pharmacol Ther 2018, 188:80-96.

4. Anfinsen KP, Grotmol T, Bruland OS, Jonasdottir TJ: Breed-specific incidence rates of canine primary bone tumors--a population based survey of dogs in Norway. Can J Vet Res 2011, 75:209-215.

5. Longhi A, Errani C, De Paolis M, Mercuri M, Bacci G: Primary bone osteosarcoma in the pediatric age: state of the art. Cancer Treat Rev 2006, 32:423-436.

6. Selmic L, Lafferty M, Kamstock D, Garner A, Ehrhart N, Worley D, Withrow S, Lana S: Outcome and prognostic factors for osteosarcoma of the maxilla, mandible, or calvarium in dogs: 183 cases (1986-2012). Journal of the American Veterinary Medical Association 2014, 245.

7. Chen X, Bahrami A, Pappo A, Easton J, Dalton J, Hedlund E, Ellison D, Shurtleff S, Wu G, Wei L, et al: Recurrent somatic structural variations contribute to tumorigenesis in pediatric osteosarcoma. Cell Rep 2014, 7:104-112.

8. Perry JA, Kiezun A, Tonzi P, Van Allen EM, Carter SL, Baca SC, Cowley GS, Bhatt AS, Rheinbay E, Pedamallu CS, et al: Complementary genomic approaches highlight the 
PI3K/mTOR pathway as a common vulnerability in osteosarcoma. Proc Natl Acad Sci U S A 2014, 111:E5564-5573.

9. Kovac M, Blattmann C, Ribi S, Smida J, Mueller NS, Engert F, Castro-Giner F, Weischenfeldt J, Kovacova M, Krieg A, et al: Exome sequencing of osteosarcoma reveals mutation signatures reminiscent of BRCA deficiency. Nat Commun 2015, 6:8940.

10. Sayles LC, Breese MR, Koehne AL, Leung SG, Lee AG, Liu HY, Spillinger A, Shah AT, Tanasa B, Straessler K, et al: Genome-Informed Targeted Therapy for Osteosarcoma. Cancer Discov 2019, 9:46-63.

11. Sakthikumar S, Elvers I, Kim J, Arendt ML, Thomas R, Turner-Maier J, Swofford R, Johnson J, Schumacher SE, Alfoldi J, et al: SETD2 Is Recurrently Mutated in WholeExome Sequenced Canine Osteosarcoma. Cancer Res 2018, 78:3421-3431.

12. Gardner HL, Sivaprakasam K, Briones N, Zismann V, Perdigones N, Drenner K, Facista $\mathrm{S}$, Richholt R, Liang W, Aldrich J, et al: Canine osteosarcoma genome sequencing identifies recurrent mutations in DMD and the histone methyltransferase gene SETD2. Commun Biol 2019, 2:266.

13. Wedekind M, Wagner L, Cripe T: Immunotherapy for osteosarcoma: Where do we go from here? Pediatric blood \& cancer 2018, 65.

14. Yu Y, Zhang H, Ren T, Huang Y, Liang X, Wang W, Niu J, Han Y, Guo W: Development of a prognostic gene signature based on an immunogenomic infiltration analysis of osteosarcoma. Journal of cellular and molecular medicine 2020, 24:11230-11242.

15. Wu C, Beird H, Andrew LJ, Advani S, Mitra A, Cao S, Reuben A, Ingram D, Wang W, $\mathrm{Ju} \mathrm{Z}$, et al: Immuno-genomic landscape of osteosarcoma. Nature communications 2020, 11.

16. Koirala P, Roth M, J G, Piperdi S, Chinai J, Geller D, Hoang B, Park A, Fremed M, Zang $\mathrm{X}$, Gorlick R: Immune infiltration and PD-L1 expression in the tumor microenvironment are prognostic in osteosarcoma. Scientific reports 2016, 6.

17. Wu C, Livingston J: Genomics and the Immune Landscape of Osteosarcoma. In Advances in experimental medicine and biology. Volume 1258. Edited by Eugenie S. Kleinerman RG: Adv Exp Med Biol; 2020: 21-36

18. Plassais J, Kim J, Davis BW, Karyadi DM, Hogan AN, Harris AC, Decker B, Parker HG, Ostrander EA: Whole genome sequencing of canids reveals genomic regions under selection and variants influencing morphology. Nat Commun 2019, 10:1489.

19. Alexandrov LB, Stratton MR: Mutational signatures: the patterns of somatic mutations hidden in cancer genomes. Curr Opin Genet Dev 2014, 24:52-60.

20. Zhao Y, Gregory M, Biertümpfel C, Hua Y, Hanaoka F, Yang W: Mechanism of somatic hypermutation at the WA motif by human DNA polymerase $\boldsymbol{\eta}$. Proceedings of the National Academy of Sciences of the United States of America 2013, 110.

21. Mermel CH, Schumacher SE, Hill B, Meyerson ML, Beroukhim R, Getz G: GISTIC2.0 facilitates sensitive and confident localization of the targets of focal somatic copynumber alteration in human cancers. In Genome Biol. Volume 12; 2011: R41

22. Ritchie ME, Phipson B, Wu D, Hu Y, Law CW, Shi W, Smyth GK: Limma powers differential expression analyses for RNA-sequencing and microarray studies. Nucleic Acids Res 2015, 43:e47. 
23. Rooney M, Shukla S, Wu C, Getz G, Hacohen N: Molecular and genetic properties of tumors associated with local immune cytolytic activity. Cell 2015, 160.

24. Newman A, Liu C, Green M, Gentles A, Feng W, Xu Y, Hoang C, Diehn M, Alizadeh A: Robust enumeration of cell subsets from tissue expression profiles. Nature methods 2015, 12.

25. Yoshihara K, Shahmoradgoli M, Martínez E, Vegesna R, Kim H, Torres-Garcia W, Treviño V, Shen H, Laird P, Levine D, et al: Inferring tumour purity and stromal and immune cell admixture from expression data. Nature communications 2013, 4.

26. Vanherberghen M, Day M, Delvaux F, Gabriel A, Clercx C, Peeters D: An immunohistochemical study of the inflammatory infiltrate associated with nasal carcinoma in dogs and cats. Journal of comparative pathology 2009, 141.

27. Chen B, Khodadoust M, Liu C, Newman A, Alizadeh A: Profiling Tumor Infiltrating Immune Cells with CIBERSORT. Methods in molecular biology (Clifton, NJ) 2018, 1711.

28. Gröbner S, Worst B, Weischenfeldt J, Buchhalter I, Kleinheinz K, Rudneva V, Johann P, Balasubramanian G, Segura-Wang M, Brabetz S, et al: The Landscape of Genomic Alterations Across Childhood Cancers. Nature 2018, 555.

29. Kirpensteijn J, Kik M, Teske E, Rutteman G: TP53 gene mutations in canine osteosarcoma. Veterinary surgery : VS 2008, 37.

30. Yokouchi H, Nishihara H, Harada T, Yamazaki S, Kikuchi H, Oizumi S, Uramoto H, Tanaka F, Harada M, Akie K, et al: Detection of somatic TP53 mutation in surgically resected small-cell lung cancer by targeted exome sequencing: association with longer relapse-free survival. Heliyon 2020, 6.

31. Esserman L, Berry D, Cheang M, Yau C, Perou C, Carey L, DeMichele A, Gray J, Conway-Dorsey $\mathrm{K}$, Lenburg $\mathrm{M}$, et al: Chemotherapy response and recurrence-free survival in neoadjuvant breast cancer depends on biomarker profiles: results from the I-SPY 1 TRIAL (CALGB 150007/150012; ACRIN 6657). Breast cancer research and treatment 2012, 132.

32. Wang Y, Y X, Chen J, Ouyang T, Li J, Wang T, Fan Z, Fan T, Lin B, Xie Y: TP53 mutations are associated with higher rates of pathologic complete response to anthracycline/cyclophosphamide-based neoadjuvant chemotherapy in operable primary breast cancer. International journal of cancer 2016, 138.

33. Chen M, Zhu Y, Xu J, Wang L, Liu C, Ji Z, Lu P: Value of TP53 status for predicting response to neoadjuvant chemotherapy in breast cancer: a meta-analysis. PloS one 2012, 7.

34. Bertheau P, Plassa F, Espié M, Turpin E, de Roquancourt A, Marty M, Lerebours F, Beuzard Y, Janin A, de Thé H: Effect of mutated TP53 on response of advanced breast cancers to high-dose chemotherapy. Lancet (London, England) 2002, 360.

35. Bertheau P, Turpin E, Rickman D, Espié M, de Reyniès A, Feugeas J, Plassa L, Soliman H, Varna M, de Roquancourt A, et al: Exquisite sensitivity of TP53 mutant and basal breast cancers to a dose-dense epirubicin-cyclophosphamide regimen. PLoS medicine $2007,4$.

36. Jackson J, Pant V, Li Q, Chang L, Quintás-Cardama A, Garza D, Tavana O, Yang P, Manshouri $\mathrm{T}$, Li $\mathrm{Y}$, et al: p53-mediated senescence impairs the apoptotic response to chemotherapy and clinical outcome in breast cancer. Cancer cell 2012, 21. 
973 37. Liu D, Song $\mathrm{H}, \mathrm{Xu} \mathrm{Y}$ : A common gain of function of p53 cancer mutants in inducing genetic instability. Oncogene 2010, 29.

38. Sottnik J, Rao S, Lafferty M, Thamm D, Morley P, Withrow S, Dow S: Association of blood monocyte and lymphocyte count and disease-free interval in dogs with osteosarcoma. Journal of veterinary internal medicine 2010, 24:1439-1444.

39. Chen R, Zhao W, Fang C, Yang X, Ji M: Histone Methyltransferase SETD2: A Potential Tumor Suppressor in Solid Cancers. Journal of Cancer 2020, 11.

40. Angstadt AY, Motsinger-Reif A, Thomas R, Kisseberth WC, Guillermo Couto C, Duval DL, Nielsen DM, Modiano JF, Breen M: Characterization of canine osteosarcoma by array comparative genomic hybridization and RT-qPCR: signatures of genomic imbalance in canine osteosarcoma parallel the human counterpart. Genes Chromosomes Cancer 2011, 50:859-874.

41. Cui J, Dean D, Hornicek FJ, Chen Z, Duan Z: The role of extracelluar matrix in osteosarcoma progression and metastasis. Journal of Experimental \& Clinical Cancer Research 2020, 39:1-11.

42. Harisi R, Dudas J, Nagy-Olah J, Timar F, Szendroi M, Jeney A: Extracellular matrix induces doxorubicin-resistance in human osteosarcoma cells by suppression of p53 function. Cancer biology \& therapy 2007, 6.

43. Chamberlain J, Metzger J, Reyes M, Townsend D, Faulkner J: Dystrophin-deficient mdx mice display a reduced life span and are susceptible to spontaneous rhabdomyosarcoma. FASEB journal : official publication of the Federation of American Societies for Experimental Biology 2007, 21.

44. Heeg S, Das K, Reicher tM, Bakir B, Takano S, Caspers J, Aiello N, Wu K, Neesse A, Maitra A, et al: ETS-Transcription Factor ETV1 Regulates Stromal Expansion and Metastasis in Pancreatic Cancer. Gastroenterology 2016, 151.

45. de Launoit Y, Chotteau-Lelievre A, Beaudoin C, Coutte L, Netzer S, Brenner C, Huvent I, Baert J: The PEA3 group of ETS-related transcription factors. Role in breast cancer metastasis. Advances in experimental medicine and biology 2000, 480.

46. Chi P, Chen Y, Zhang L, Guo X, Wongvipat J, Shamu T, Fletcher JA, Dewell S, Maki RG, Zheng D, et al: ETV1 is a lineage survival factor that cooperates with KIT in gastrointestinal stromal tumours. Nature 2010, 467:849-853.

47. Li Z, Zhang L, Ma Z, Yang M, Tang J, Fu Y, Mao Y, Hong X, Zhang Y: ETV1 induces epithelial to mesenchymal transition in human gastric cancer cells through the upregulation of Snail expression. Oncology reports 2013, 30.

48. Bailey J, Alsina J, Rasheed Z, McAllister F, Fu Y, Plentz R, Zhang H, Pasricha P, Bardeesy N, Matsui W, et al: DCLK1 marks a morphologically distinct subpopulation of cells with stem cell properties in preinvasive pancreatic cancer. Gastroenterology 2014, 146.

49. Maniati E, Bossard M, Cook N, Candido J, Emami-Shahr iN, Nedospasov S, Balkwill F, Tuveson D, Hagemann T: Crosstalk between the canonical NF-кB and Notch signaling pathways inhibits Ppary expression and promotes pancreatic cancer progression in mice. The Journal of clinical investigation 2011, 121.

50. Slot A, Molinski S, Cole S: Mammalian multidrug-resistance proteins (MRPs). Essays in biochemistry 2011, 50:179-207. 
51. DeNicola G, Karreth F, Humpton T, Gopinathan A, Wei C, Frese K, Mangal D, Yu K, Yeo C, Calhoun E, et al: Oncogene-induced Nrf2 transcription promotes ROS

\section{detoxification and tumorigenesis. Nature 2011, 475.}

52. Panieri E, Saso L: Potential Applications of NRF2 Inhibitors in Cancer Therapy. Oxidative medicine and cellular longevity 2019, 2019.

53. Robert C, Ribas A, Schachter J, Arance A, Grob J, Mortier L, Daud A, Carlino M, McNeil $C$, Lotem $M$, et al: Pembrolizumab versus ipilimumab in advanced melanoma (KEYNOTE-006): post-hoc 5-year results from an open-label, multicentre, randomised, controlled, phase 3 study. The Lancet Oncology 2019, 20.

54. Motzer R, Escudier B, McDermott D, George S, Hammers H, Srinivas S, Tykodi S, Sosman J, Procopio G, Plimack E, et al: Nivolumab versus Everolimus in Advanced Renal-Cell Carcinoma. The New England journal of medicine 2015, 373.

55. Bellmunt J, de Wit R, Vaughn D, Fradet Y, Lee J, Fong L, Vogelzang N, Climent M, Petrylak D, Choueiri T, et al: Pembrolizumab as Second-Line Therapy for Advanced Urothelial Carcinoma. The New England journal of medicine 2017, 376.

56. Le D, Uram J, Wang H, Bartlett B, Kemberling H, Eyring A, Skora A, Luber B, Azad N, Laheru D, et al: PD-1 Blockade in Tumors with Mismatch-Repair Deficiency. The New England journal of medicine 2015, 372.

57. Ferris R, Blumenschein G, Fayette J, Guigay J, Colevas A, Licitra L, Harrington K, Kasper S, Vokes E, Even C, et al: Nivolumab for Recurrent Squamous-Cell Carcinoma of the Head and Neck. The New England journal of medicine 2016, 375.

58. Behjati S, Tarpey P, Haase K, Ye H, Young M, Alexandrov L, Farndon S, Collord G, Wedge D, Martincorena I, et al: Recurrent mutation of IGF signalling genes and distinct patterns of genomic rearrangement in osteosarcoma. Nature communications 2017,8 .

59. Tawbi H, Burgess M, Bolejack V, Van Tine B, Schuetze S, Hu J, D'Angelo S, Attia S, Riedel R, Priebat D, et al: Pembrolizumab in advanced soft-tissue sarcoma and bone sarcoma (SARC028): a multicentre, two-cohort, single-arm, open-label, phase 2 trial. The Lancet Oncology 2017, 18.

60. D'Angelo S, Mahoney M, Van Tine B, Atkins J, Milhem M, Jahagirdar B, Antonescu C, Horvath E, Tap W, Schwartz G, Streicher H: Nivolumab with or without ipilimumab treatment for metastatic sarcoma (Alliance A091401): two open-label, noncomparative, randomised, phase 2 trials. The Lancet Oncology 2018, 19.

61. Meyers P: Muramyl Tripeptide-Phosphatidyl Ethanolamine Encapsulated in Liposomes (L-MTP-PE) in the Treatment of Osteosarcoma. In Current Advances in Osteosarcoma. Volume 1257: Adv Exp Med Biol; 2020: 133-139

62. Meyers P, Schwartz C, Krailo M, Healey J, Bernstein M, Ferguson W, Gebhardt M, Goorin A, Harris M, Kleinerman E, et al: Osteosarcoma: the addition of muramyl tripeptide to chemotherapy improves overall survival--a report from the Children's Oncology Group. Journal of clinical oncology : official journal of the American Society of Clinical Oncology 2008, 26.

63. Paoloni M, Davis S, Lana S, Withrow S, Sangiorgi L, Picci P, Hewitt S, Triche T, Meltzer $P$, Khanna $C$ : Canine tumor cross-species genomics uncovers targets linked to osteosarcoma progression. BMC genomics 2009, 10.

64. Fan T, Khanna C: Comparative Aspects of Osteosarcoma Pathogenesis in Humans and Dogs. Veterinary sciences 2015, 2. 
65. Scott M, Temiz N, Sarver A, LaRue R, Rathe S, Varshney J, Wolf N, Moriarity B, O'Brien T, Spector L, et al: Comparative Transcriptome Analysis Quantifies Immune Cell Transcript Levels, Metastatic Progression, and Survival in Osteosarcoma. Cancer research 2018, 78.

66. Zaravinos A, Roufas C, Nagara M, de Lucas Moreno B, Oblovatskaya M, Efstathiades C, Dimopoulos C, Ayiomamitis G: Cytolytic activity correlates with the mutational burden and deregulated expression of immune checkpoints in colorectal cancer. Journal of experimental \& clinical cancer research : CR 2019, 38:364.

67. Bolger AM, Lohse M, Usadel B: Trimmomatic: a flexible trimmer for Illumina sequence data. Bioinformatics 2014, 30:2114-2120.

68. Andrews S: FastQC A Quality Control tool for High Throughput Sequence Data ( https://www.bioinformatics.babraham.ac.uk/projects/fastqc/). 2010.

69. Li H, Durbin R: Fast and accurate long-read alignment with Burrows-Wheeler transform. Bioinformatics 2010, 26:589-595.

70. Van der Auwera GA, Carneiro MO, Hartl C, Poplin R, Del Angel G, Levy-Moonshine A, Jordan T, Shakir K, Roazen D, Thibault J, et al: From FastQ data to high confidence variant calls: the Genome Analysis Toolkit best practices pipeline. Curr Protoc Bioinformatics 2013, 43:11.10.11-33.

71. Blokzijl F, Janssen R, van Boxtel R, Cuppen E: MutationalPatterns: comprehensive genome-wide analysis of mutational processes. Genome medicine 2018, 10.

72. Tate J, Bamford S, Jubb H, Sondka Z, Beare D, Bindal N, Boutselakis H, Cole C, Creatore C, Dawson E, et al: COSMIC: the Catalogue Of Somatic Mutations In Cancer. Nucleic acids research 2019, 47.

73. Huang da W, Sherman BT, Zheng X, Yang J, Imamichi T, Stephens R, Lempicki RA: Extracting biological meaning from large gene lists with DAVID. Curr Protoc Bioinformatics 2009, Chapter 13:Unit 13.11.

74. Chen E, Tan C, Kou Y, Duan Q, Wang Z, Meirelles G, Clark N, Ma'ayan A: Enrichr: interactive and collaborative HTML5 gene list enrichment analysis tool. $B M C$ bioinformatics 2013, 14.

75. Favero F, Joshi T, Marquard AM, Birkbak NJ, Krzystanek M, Li Q, Szallasi Z, Eklund AC: Sequenza: allele-specific copy number and mutation profiles from tumor sequencing data. Ann Oncol 2015, 26:64-70.

76. Subramanian A, Tamayo P, Mootha V, Mukherjee S, Ebert B, Gillette M, Paulovich A, Pomeroy S, Golub T, Lander E, Mesirov J: Gene set enrichment analysis: a knowledge-based approach for interpreting genome-wide expression profiles. Proceedings of the National Academy of Sciences of the United States of America 2005, 102.

77. Liberzon A, Subramanian A, Pinchback R, Thorvaldsdóttir H, Tamayo P, JP M: Molecular signatures database (MSigDB) 3.0. Bioinformatics (Oxford, England) 2011, 27.

78. R: The R Project for Statistical Computing. [https://www.r-project.org/]

79. Anderson J, Burns PJ, Milroy D, Ruprecht P, Hauser T, Siegel HJ: Deploying RMACC Summit: An HPC Resource for the Rocky Mountain Region. In Proceedings of the Practice and Experience in Advanced Research Computing 2017 on Sustainability, Success and Impact; 07/09/2017. ACM; 2017: 8. 
1111 Figures.

Fig. 1
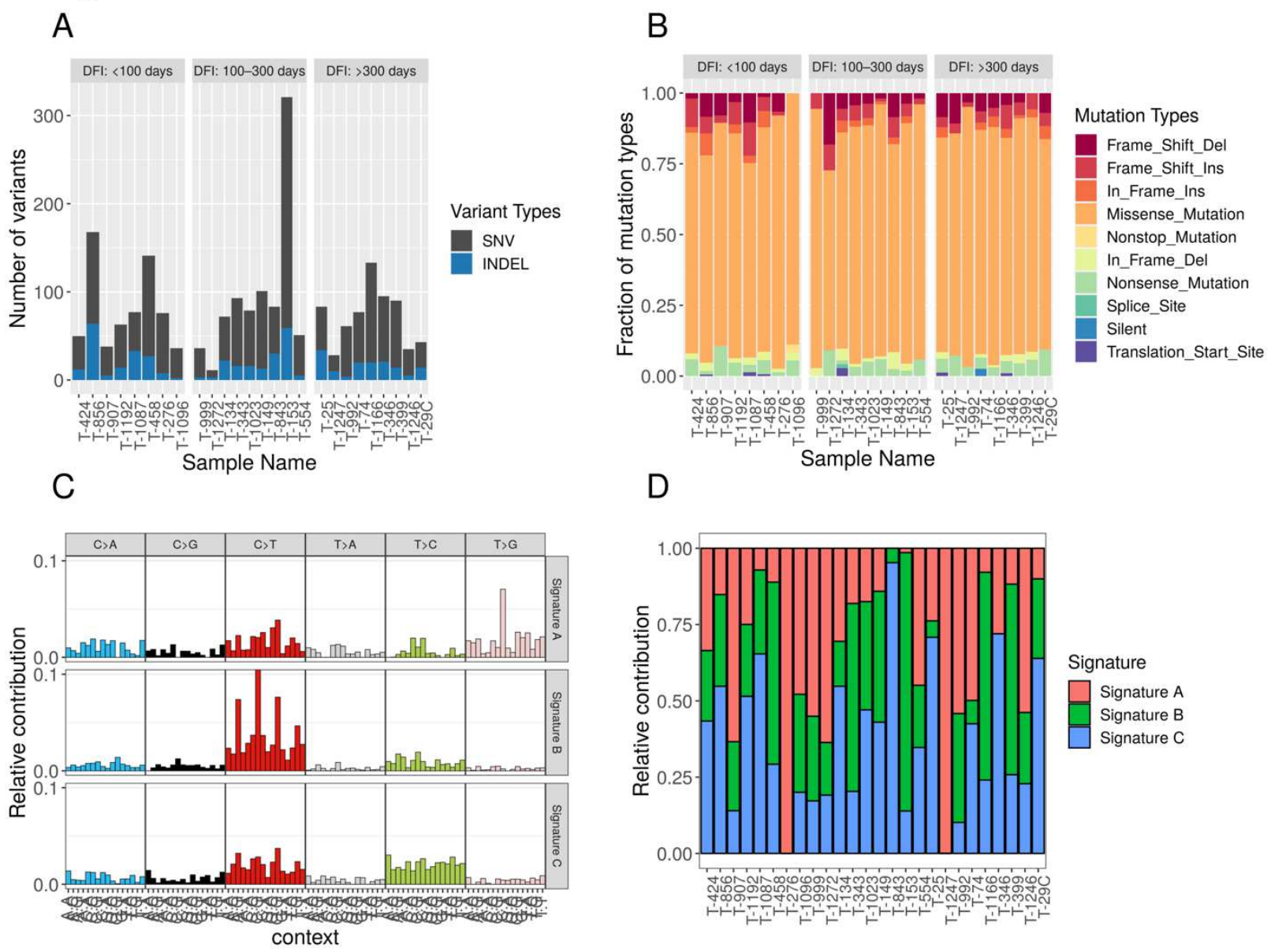

1114 Fig. 1. Mutational signature profile in canine osteosarcoma. A. Distribution of total short variants (SNVs and INDELs) across 26 samples. The samples were sorted in ascending order of disease-free interval (DFI) and binned in three discrete groups. B. Distribution of mutation types as identified by VEP across 26 samples. Missense mutations were the most common type of short variant in this dataset. C. Mutation signature analyses showing the relative distribution of six single nucleotide changes in 96 different trinucleotide contexts across three de novo signatures. D. Relative contribution of three predominant signatures across 26 samples. 


\section{Fig. 2}

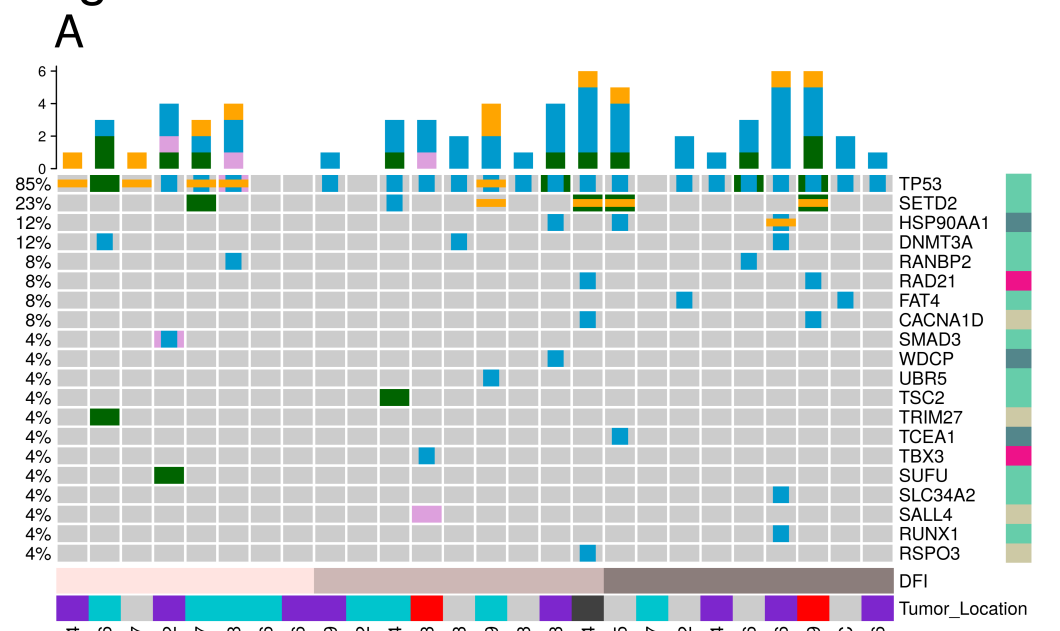

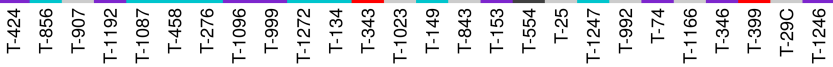

\begin{tabular}{|c|c|c|c|c|}
\hline \multicolumn{2}{|c|}{ MutationType } & DFI & Tumor Location & CancerGeneCensus \\
\hline $\begin{array}{l}\text { Missense } \\
\text { Frameshift Deletion }\end{array}$ & $\begin{array}{l}\text { Frameshift Insertion } \\
\text { Stop gained }\end{array}$ & $\begin{array}{l}<100 \text { days } \\
>300 \text { days } \\
100 \text { to } 300 \text { days }\end{array}$ & $\begin{array}{l}\text { Femur } \\
\text { Humerus } \\
\text { Radius } \\
\text { Rcapula } \\
\text { Sibia }\end{array}$ & $\begin{array}{l}\text { Both } \\
\text { Oncogene } \\
\text { TSG } \\
\text { Unassigned }\end{array}$ \\
\hline
\end{tabular}

B

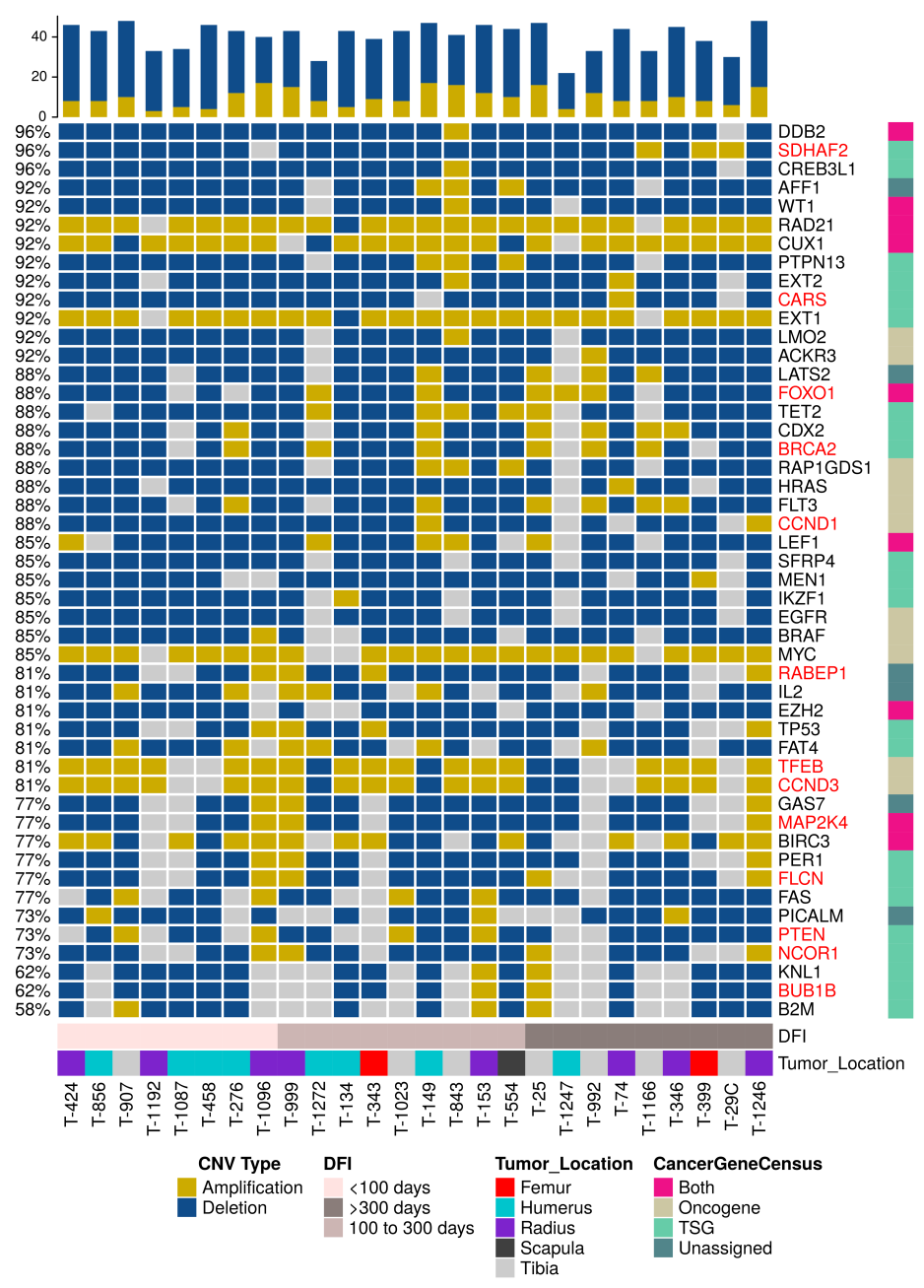

Fig. 2. Oncoplot of cancer census genes with variants in canine osteosarcoma samples. A. The genes with short variants (SNVs and INDELs) are plotted along with sample characteristics like disease free interval (DFI) and tumor location. The cancer genes were categorized as oncogenes and tumor suppressor genes in accordance to the Cancer Gene Census. B. The genes with recurrent copy number variations (amplifications and deletions) were plotted. The samples were sorted in ascending order of DFI in both plots. Gene names colored red indicate significant correlation of copy number amplitude and transcript expression. 


\section{Fig. 3}

A

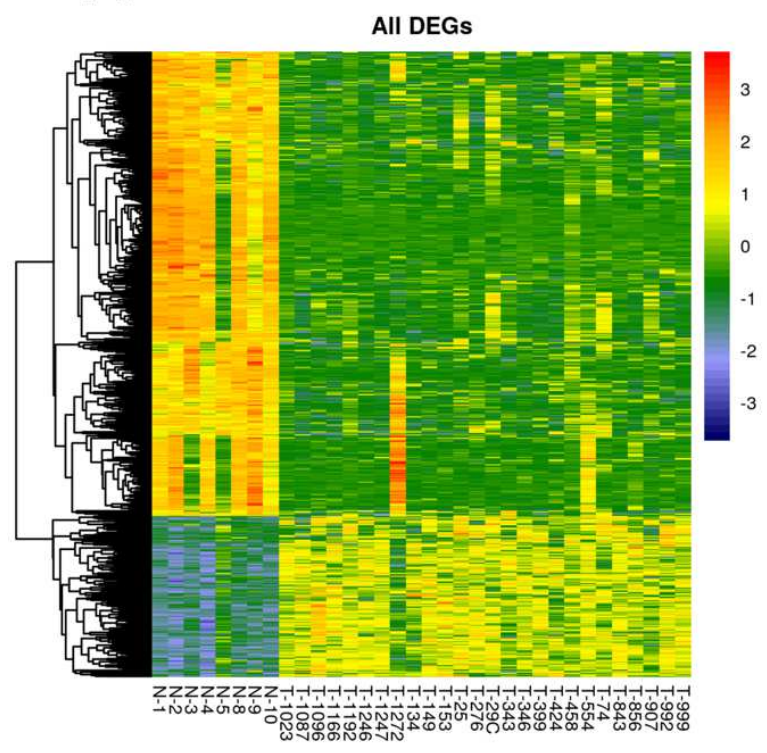

C

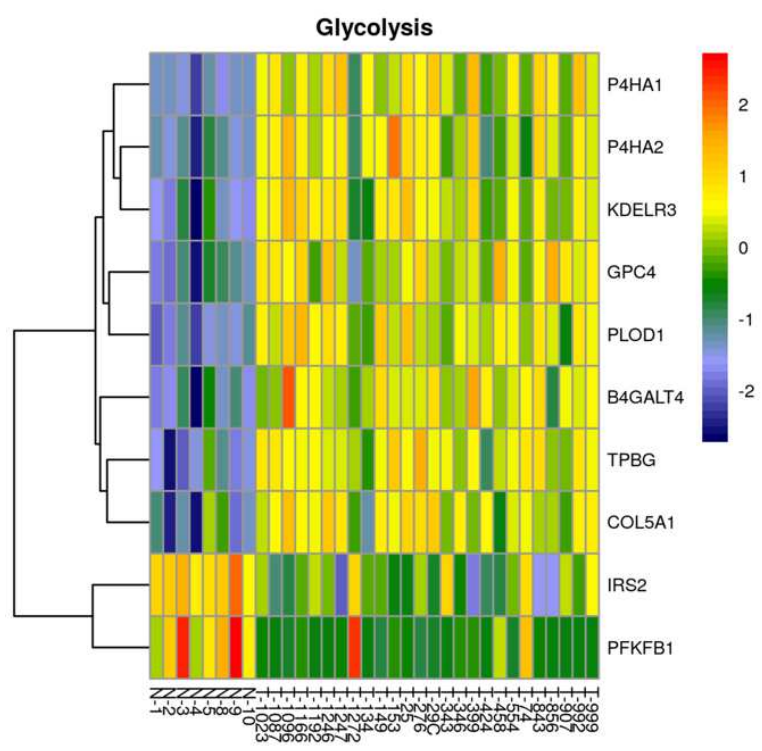

B
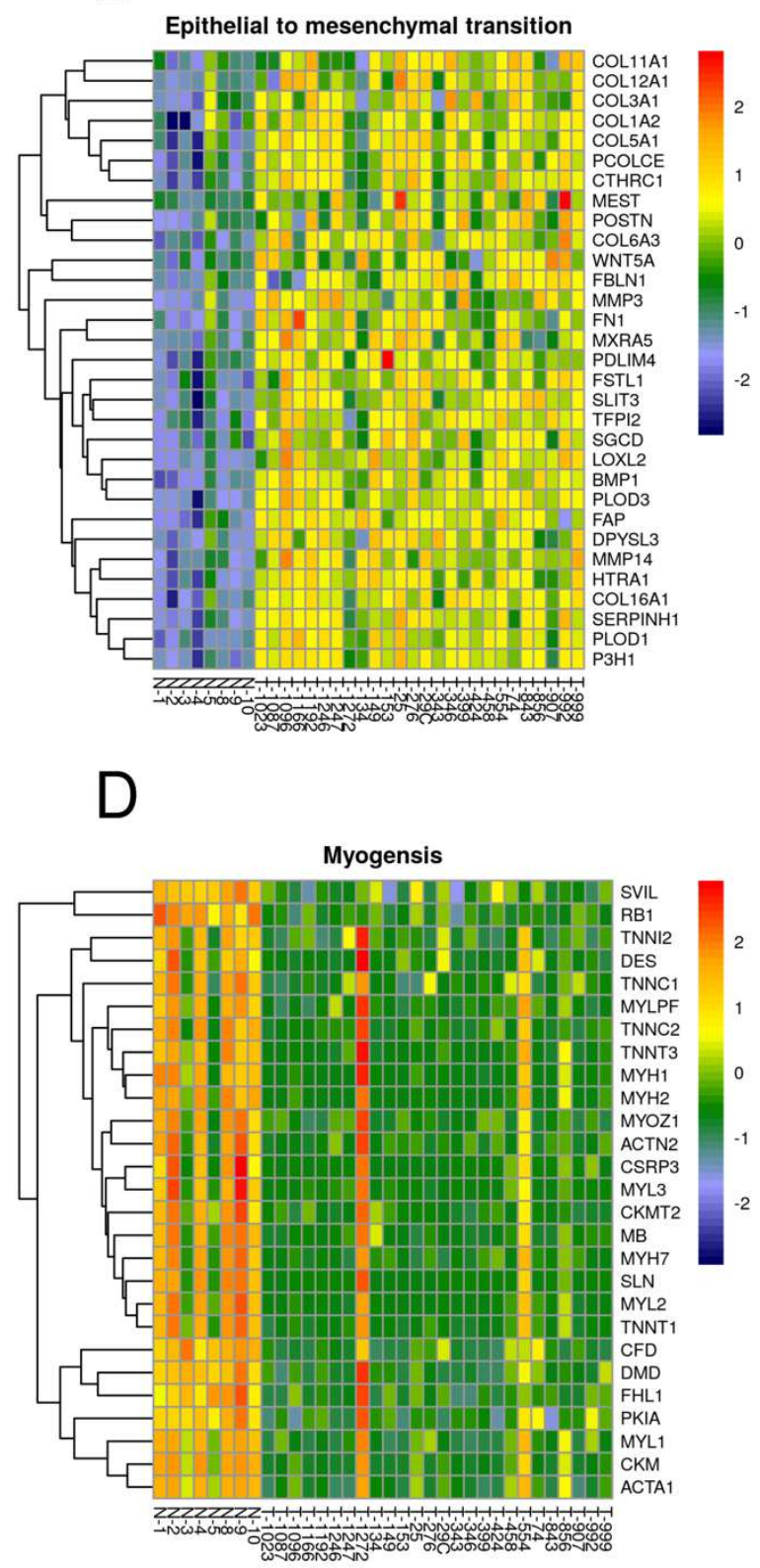

1155 Fig.3. Graphical representation of differentially expressed genes (DEG). A. Heatmap of DEGs 1156 between normal bone ( $\mathrm{N}-$ ) and canine osteosarcoma samples (T-). B-D. The heatmap of core1157 enriched genes associated with three significantly enriched Hallmark gene sets in tumor 1158 samples in comparison to normal bone, as identified by GSEA analysis. 


\section{Fig. 4}

\section{A}
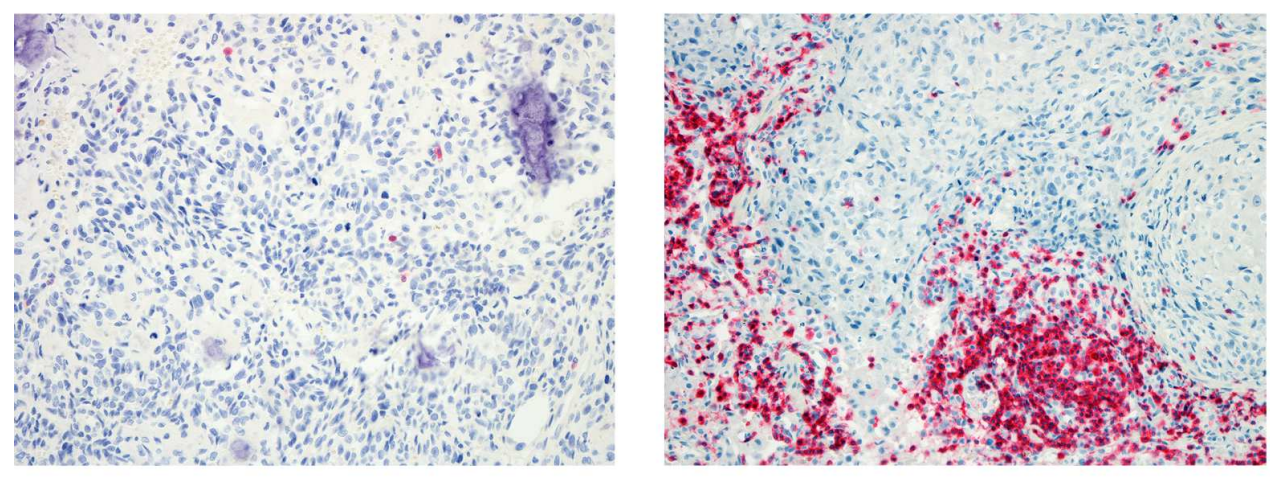

C
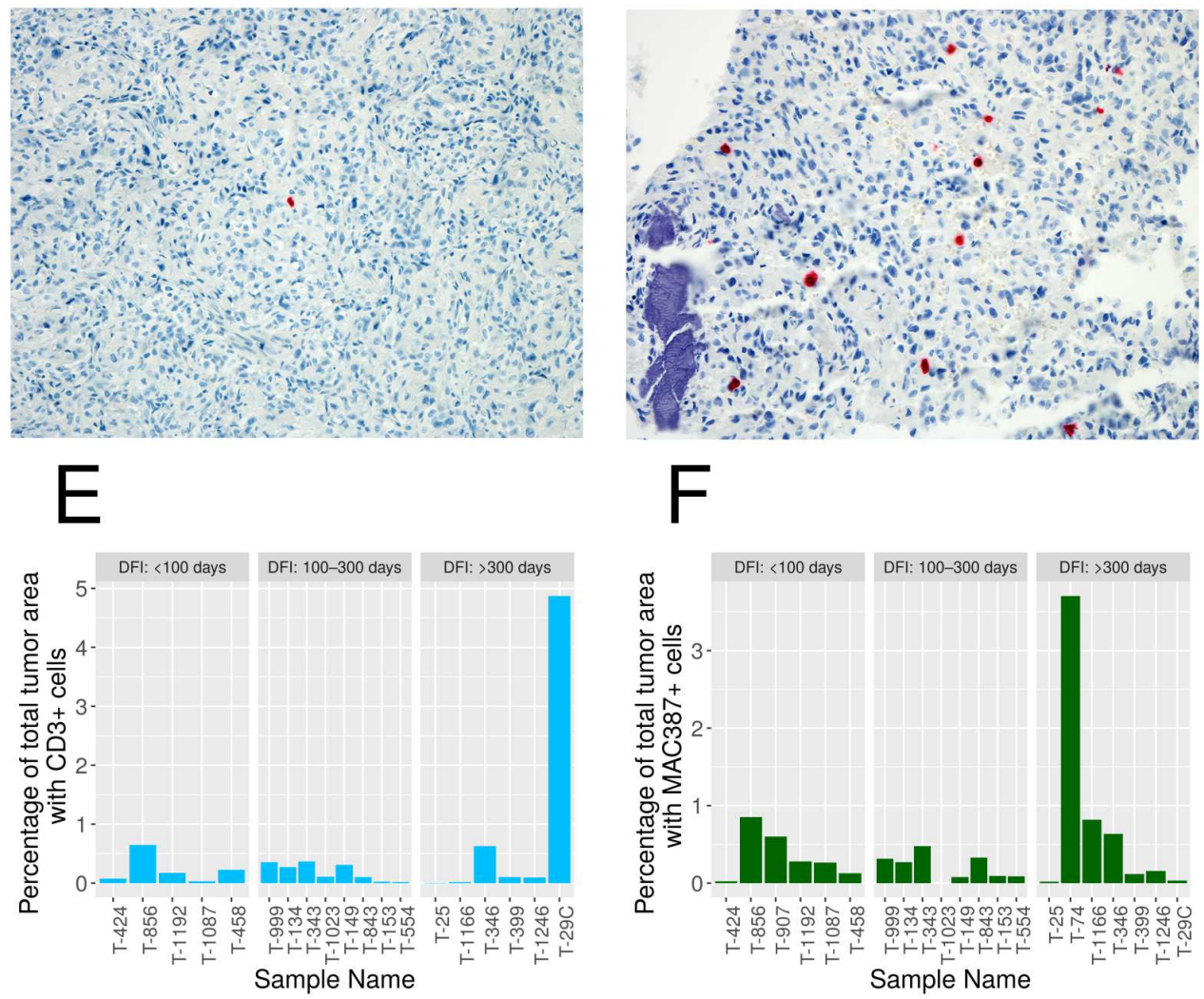

1179 Fig.4. Association of TP53 mutation status and clinical parameters with outcome. A. Kaplan 1180 Meier plots for disease free interval (DFI) in dogs with osteosarcoma stratified by TP53 1181 mutation status. The dogs with wild type/null TP53 status had a shorter DFI compared to dogs 1182 with TP53 missense mutations. B. Kaplan Meier plots for dogs with osteosarcoma stratified by 1183 pre-treatment monocyte count ( $>$ or $<0.04 * 10^{3}$ cells $/ \mu \mathrm{l}$ ). Higher monocyte counts were 1184 associated with short DFI patients. 
Fig. 5

A

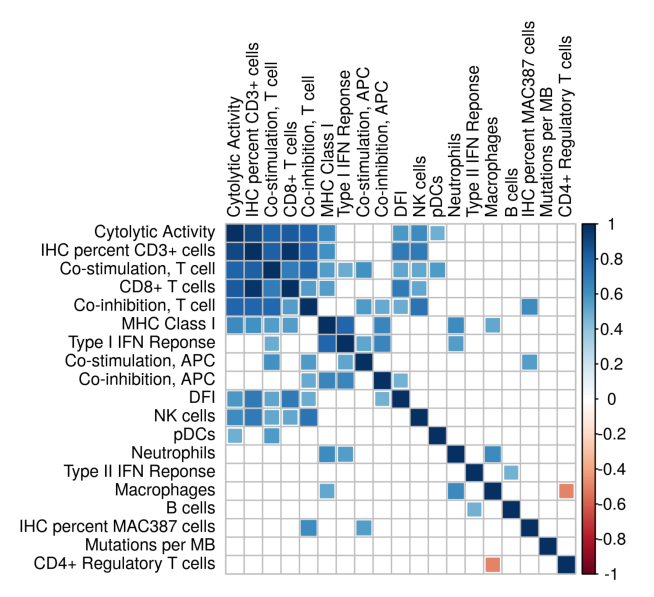

B

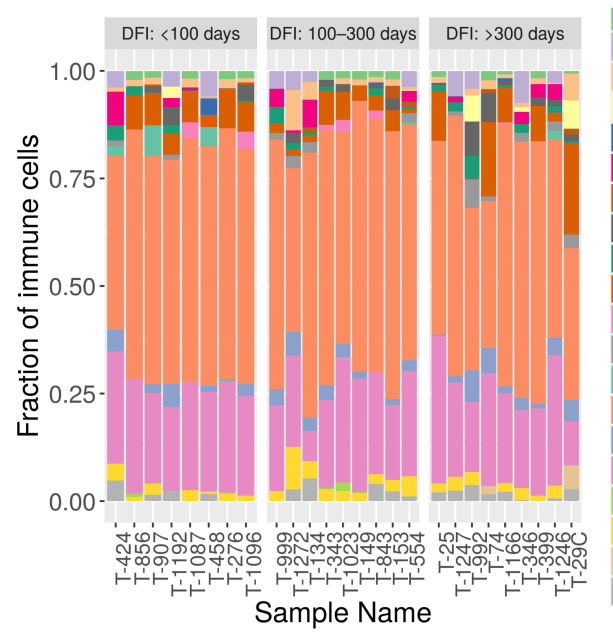

$B$ cells naive B cells memory Plasma cells T cells CD8 $T$ cells CD4 naive T cells CD4 memory resting T cells CD4 memory activated $T$ cells follicular helper $T$ cells regulatory (Tregs) $T$ cells gamma delta NK cells resting NK cells activated Monocytes Macrophages M0 Macrophages M1 Macrophages M2 Dendritic cells activated Mast cells resting Mast cells activated Neutrophils

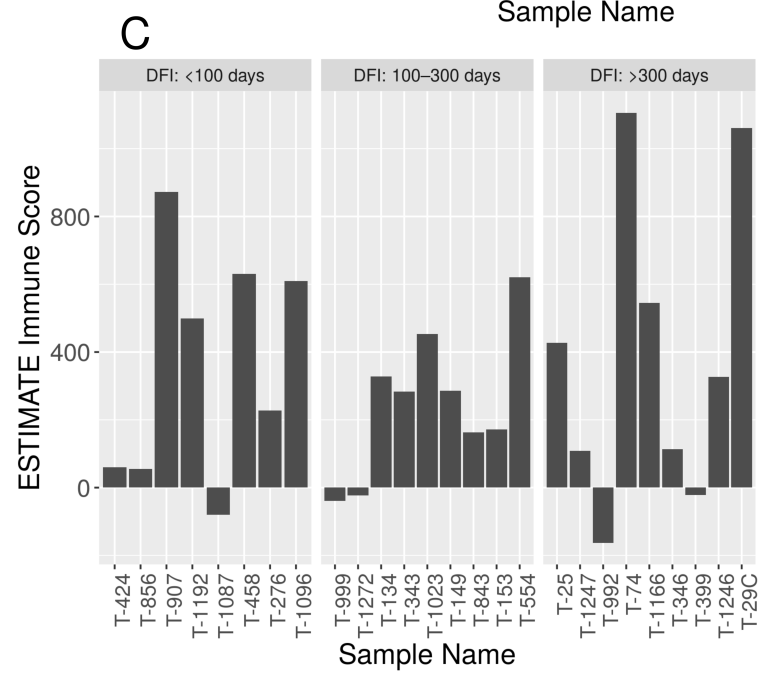

Fig 5. Representative figures of immunohistochemical staining of canine osteosarcoma samples. A-B. These samples were stained for CD3+ T-cell infiltration in A (low levels - T-1023) and B (high levels - T-1162). C-D. These samples were stained with the MAC387 antibody against S100A9 to distinguish macrophage infiltration. C represents low levels (T-1162) and D represents high levels (T-856). E-F. Quantification of immunohistochemical labelling of T-cell (CD3+ cells) and Macrophages (MAC387 cells) across 19 and 22 samples, respectively. 
Fig. 6
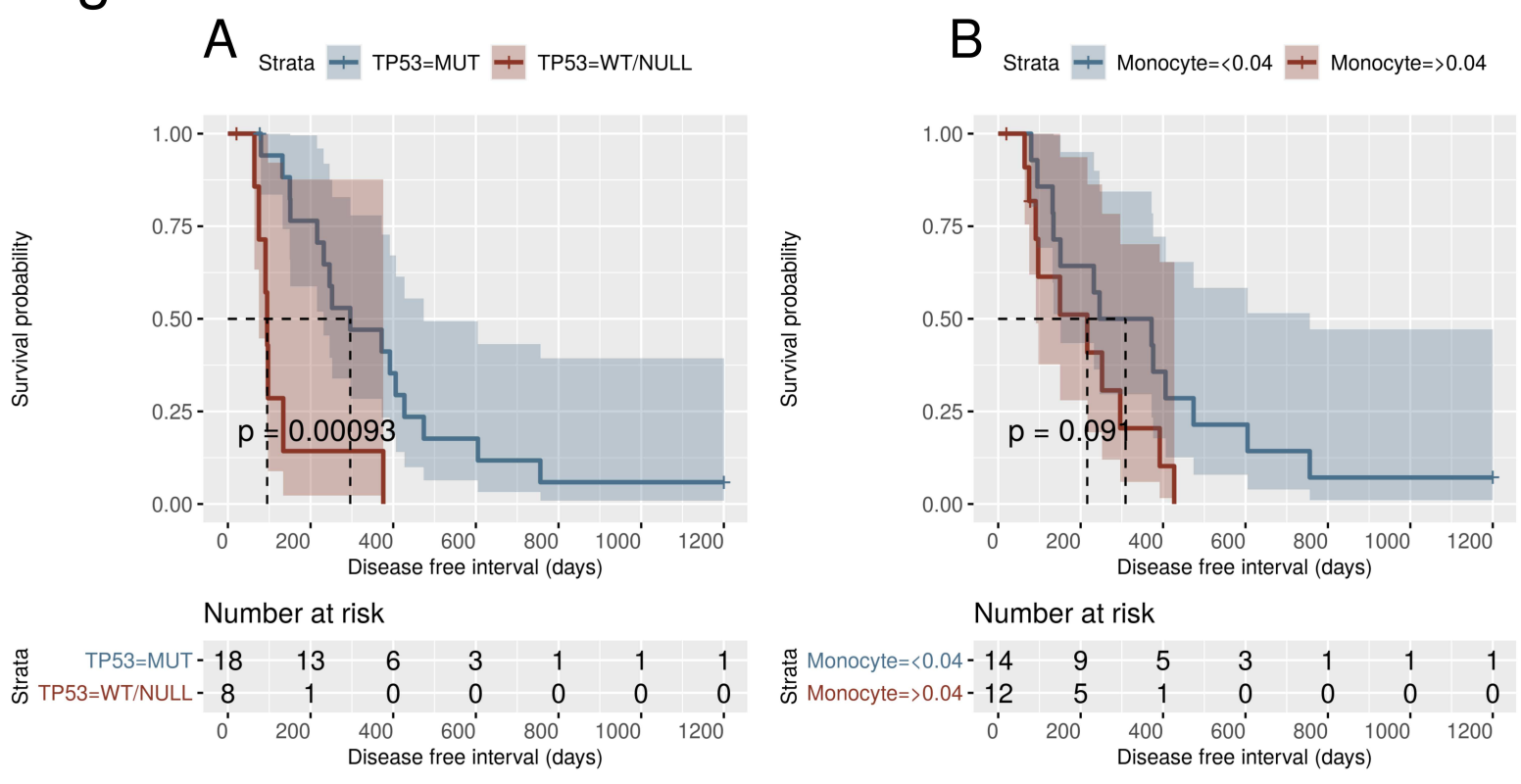

Fig.6. Profiling of immune cell infiltration using gene expression data. A. Pearson correlation matrix of immune cell expression score and immune staining levels of T-cell (IHC percent CD3+ cells) and macrophages (IHC percent MAC387 cells). We also correlated mutations per megabases (MB) and disease-free interval (DFI) with other variables. Only the significant (FDR $<0.05)$ correlation values are plotted. B. Quantification of 22 immune cell types using CIBERSORT. The samples are sorted by DFI. The most prevalent cell type in the tumor microenvironment were $\mathrm{M} 0$ and $\mathrm{M} 2$ macrophages. $\mathrm{C}$. Immune score quantification using ESTIMATE R package. 


\section{DECLARATIONS}

\section{Ethics approval and consent to participate}

1217 The animal study was reviewed and approved by Colorado State University Animal Care and

1218 Use Committee. Written informed consent was obtained from the owners for the participation

1219 of their animals in this study.

1220 Funding

1221 Funding was provided by Anschutz Foundation (DLD, DLG and DHT), P30 CA046934 (University

1222 of Colorado Cancer Center Support Grant, Genomics and Microarray Shared Resource and DL

1223 Gustafson). Funding was also provided by COXEN Retrospective Grant: Morris Animal

1224 Foundation D13CA-044 (PI: DL Gustafson) and COXEN Prospective Grant: Morris Animal

1225 Foundation D16CA-003 (PI: DL Gustafson). Additional funding includes The National Institutes

1226 of Health, Office of the Director, award number K01OD022982 (PI: DP Regan) and National

1227 Center for Advancing Translational Sciences, award number L30 TR002126 (PI: DP Regan).

1228 Acknowledgements

1229 We thank Tyler Eike for IT support and software installation. We would also like to thank

1230 Keegan Collins for prepping tumor samples, Liza Pfaff for prepping normal bone samples, and

1231 Irene Mok for her help with sample curation. The authors gratefully acknowledge the efforts of

1232 Mary Lafferty for collection of clinical outcome data. 


\section{Competing interests}

1234 There are no competing interests.

\section{Availability of data and materials}

1238 The Illumina raw fastq files were submitted to NCBI Short Read Archive (SRA) database. The

1239 canine osteosarcoma and normal samples have been submitted to Bioproject PRJNA613479

1240 and PRJNA503860, respectively. The microarray data can be downloaded from Gene Expression

1241 Omnibus (GEO) database using accession number GSE76127. The tools, databases, and their

1242 versions, in addition to the bioinformatic pipeline used in this study can be accessed here:

1243 https://github.com/sdas2019/Canine-Osteosarcoma-Whole-Exome-Sequencing-Pipeline.

\section{Author contribution}

1245 Sunetra Das: Contributed towards design of the work, analysis and interpretation of data,

1246 drafting of the manuscript.

1247 Rupa Idate: Contributed towards sample preparation used for whole exome sequencing.

1248 Daniel P. Regan: Contributed towards immunohistochemical sample processing and drafting

1249 the manuscript.

1250 Jared S. Fowles: Contributed towards microarray sample processing.

1251 Susan E. Lana: Contributed towards curations of tumor samples and is the Director of FACC 1252 tissue archive. 
1253 Douglas H. Thamm: Contributed towards analysis and interpretation of survival analysis and 1254 revision of the draft.

1255 Daniel L. Gustafson: Contributed towards providing resources for gene expression analysis and 1256 interpretation.

1257 Dawn L. Duval: Contributed towards conception and design of the work, interpretation of data, 1258 and drafting and revisions of the manuscript. 
A
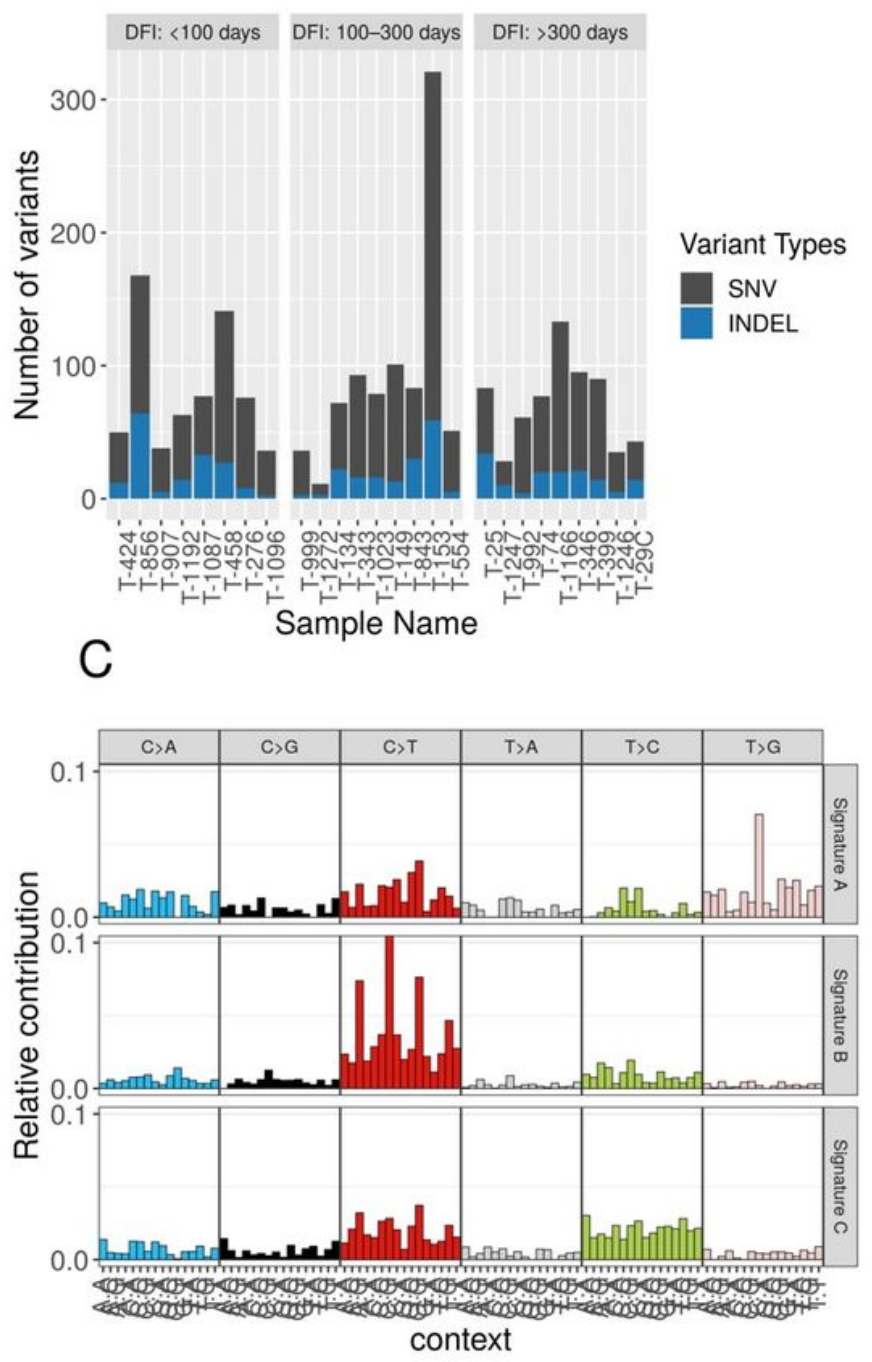

$\mathrm{B}$
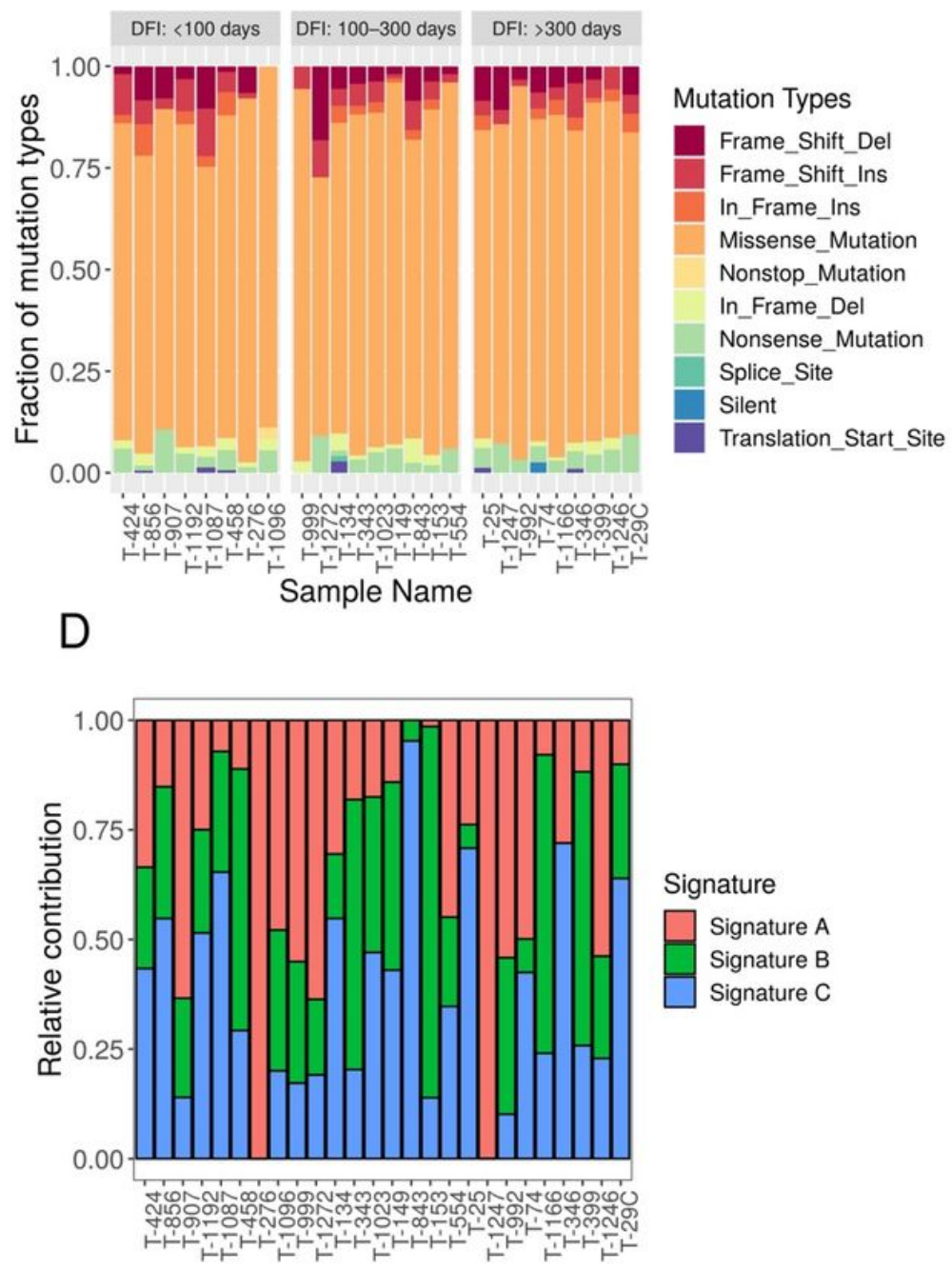

Figure 1

Mutational signature profile in canine osteosarcoma. A. Distribution of total short variants (SNVs and INDELs) across 26 samples. The samples were sorted in ascending order of disease-free interval (DFI) and binned in three discrete groups. B. Distribution of mutation types as identified by VEP across 26 samples. Missense mutations were the most common type of short variant in this dataset. C. Mutation signature analyses showing the relative distribution of six single nucleotide changes in 96 different trinucleotide contexts across three de novo signatures. D. Relative contribution of three predominant signatures across 26 samples. 


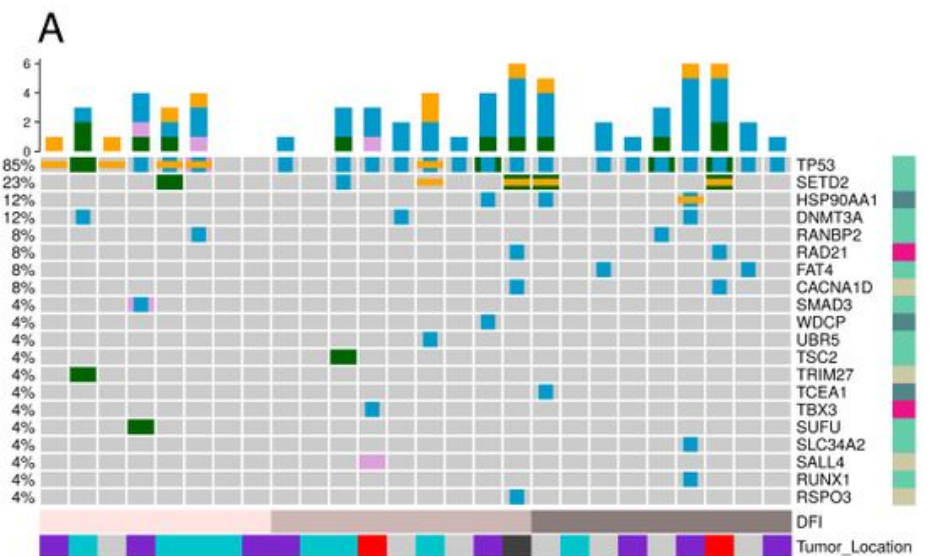

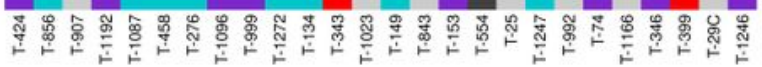

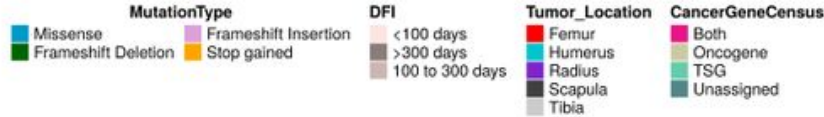

B
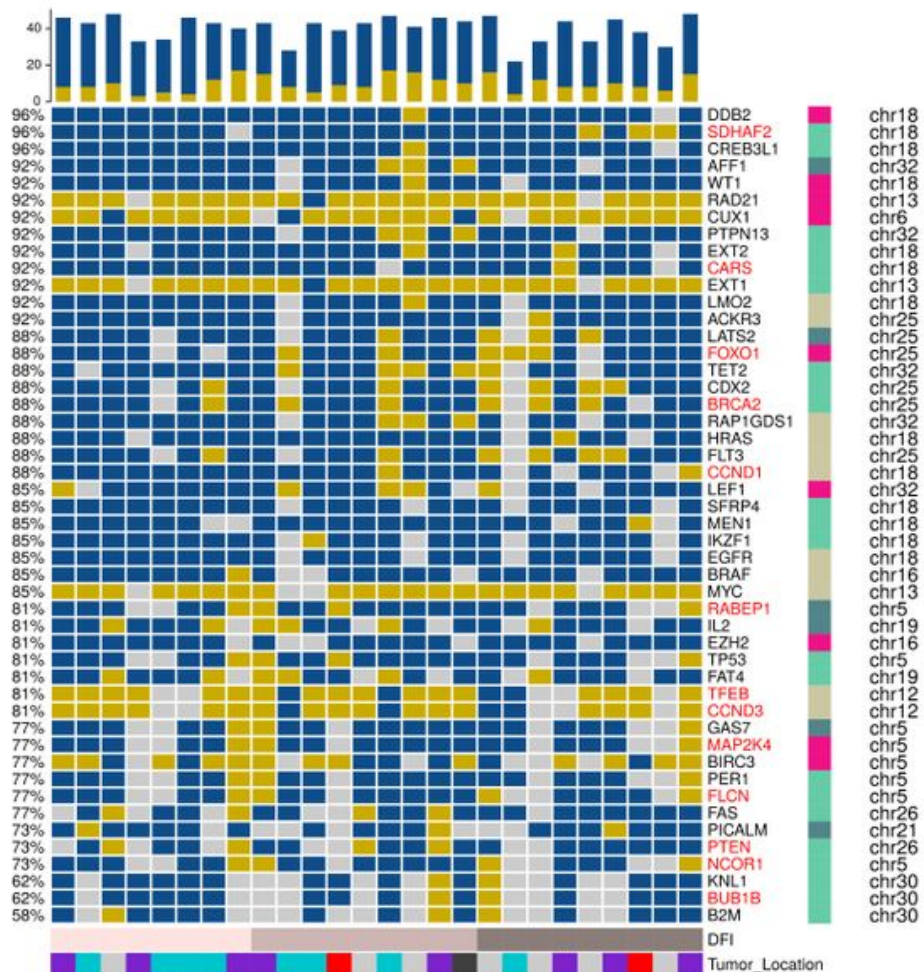

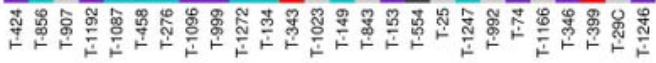

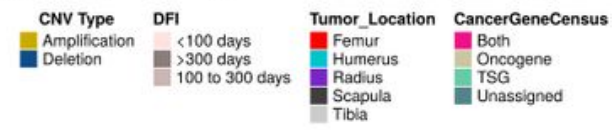

Figure 2

Oncoplot of cancer census genes with variants in canine osteosarcoma samples. A. The genes with short variants (SNVs and INDELs) are plotted along with sample characteristics like disease free interval (DFI) and tumor location. The cancer genes were categorized as oncogenes and tumor suppressor genes in accordance to the Cancer Gene Census. B. The genes with recurrent copy number variations (amplifications and deletions) were plotted. The samples were sorted in ascending order of DFI in both 
plots. Gene names colored red indicate significant correlation of copy number amplitude and transcript expression.
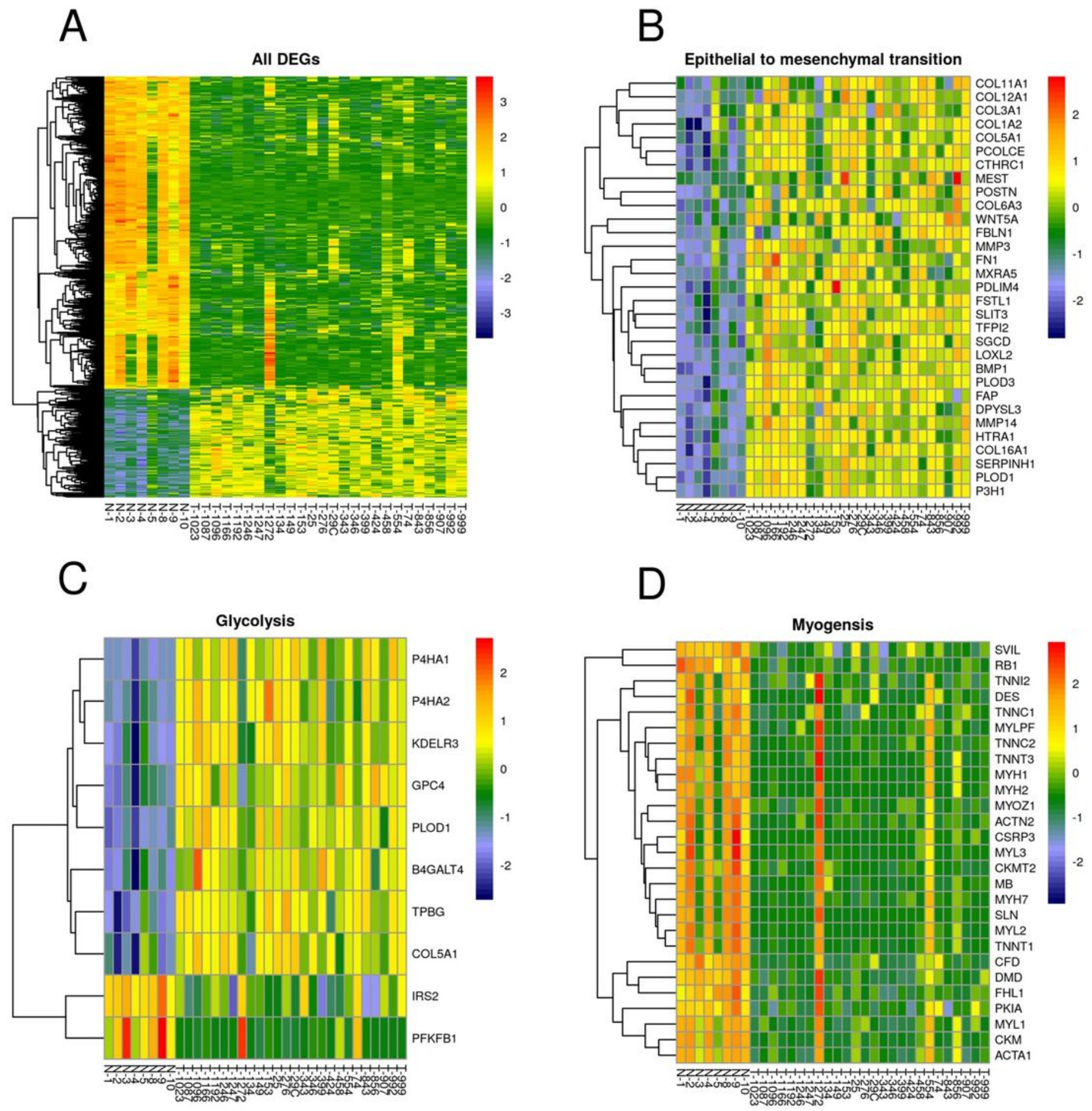

Figure 3

Graphical representation of differentially expressed genes (DEG). A. Heatmap of DEGs between normal bone $(\mathrm{N}-)$ and canine osteosarcoma samples (T-). B-D. The heatmap of core enriched genes associated 
with three significantly enriched Hallmark gene sets in tumor samples in comparison to normal bone, as identified by GSEA analysis.
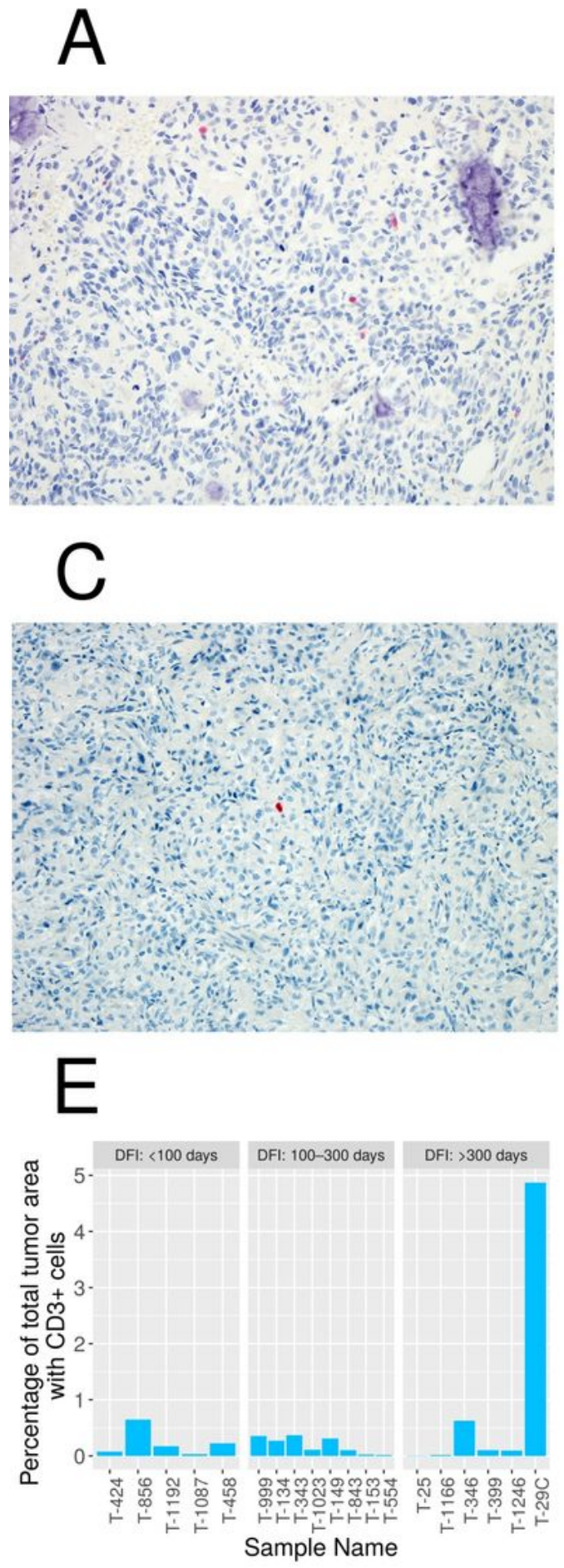
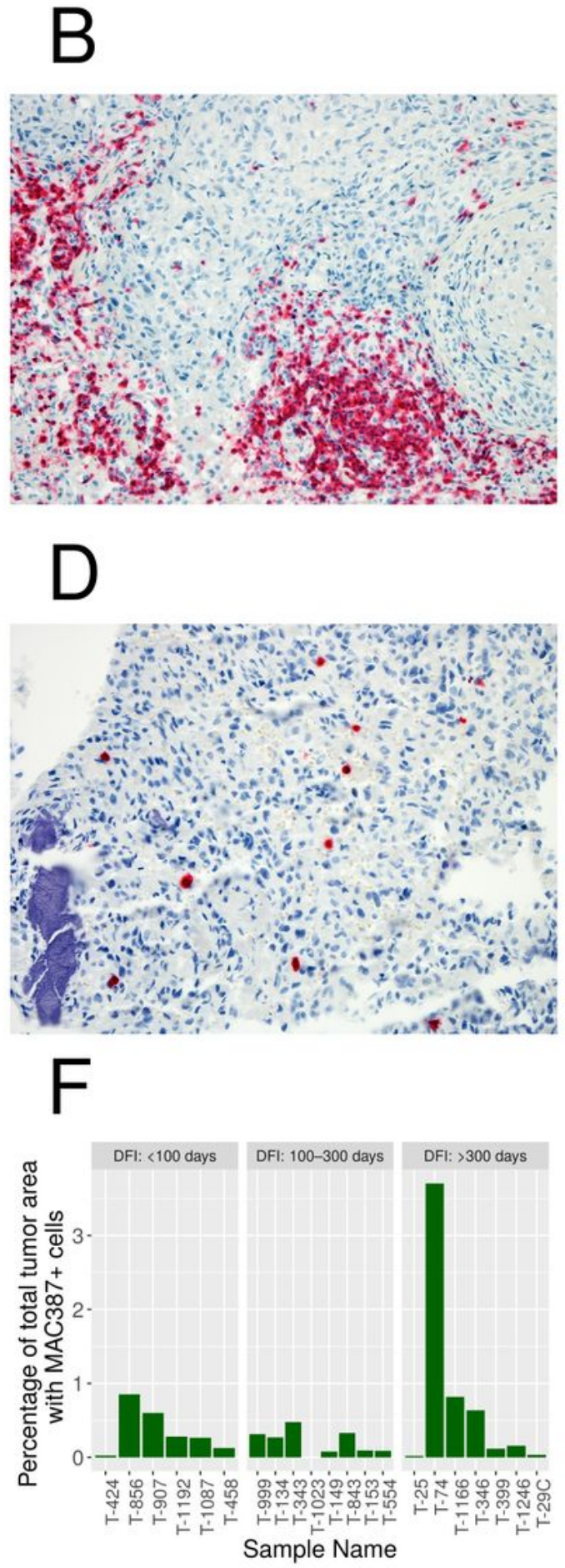

Figure 4

Association of TP53 mutation status and clinical parameters with outcome. A. Kaplan Meier plots for disease free interval (DFI) in dogs with osteosarcoma stratified by TP53 mutation status. The dogs with wild type/null TP53 status had a shorter DFI compared to dogs with TP53 missense mutations. B. Kaplan 
Meier plots for dogs with osteosarcoma stratified by pre-treatment monocyte count ( $>$ or $<0.04 * 103$ cells/ $\mu \mathrm{l})$. Higher monocyte counts were associated with short DFI patients.
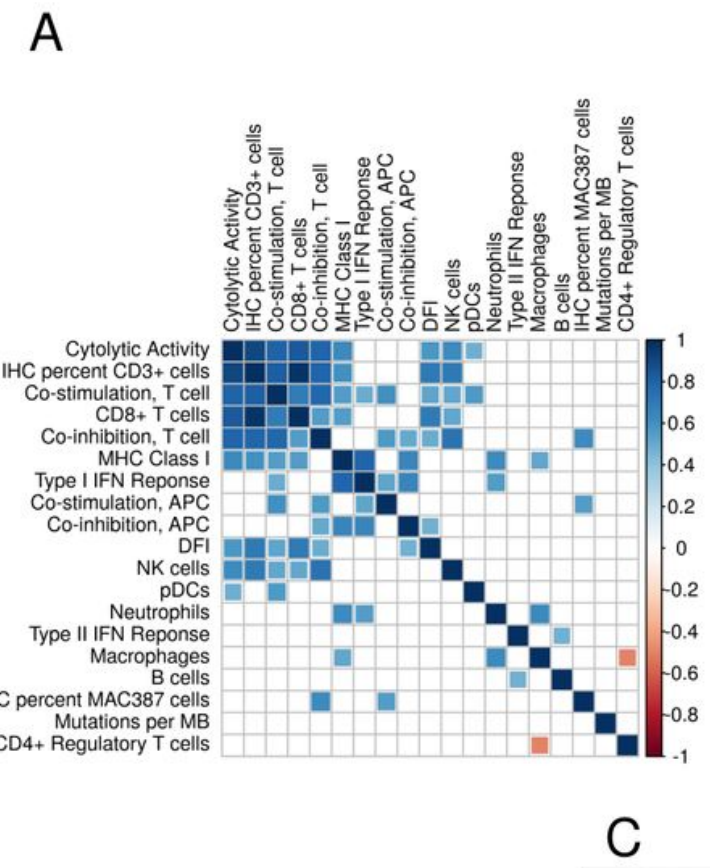

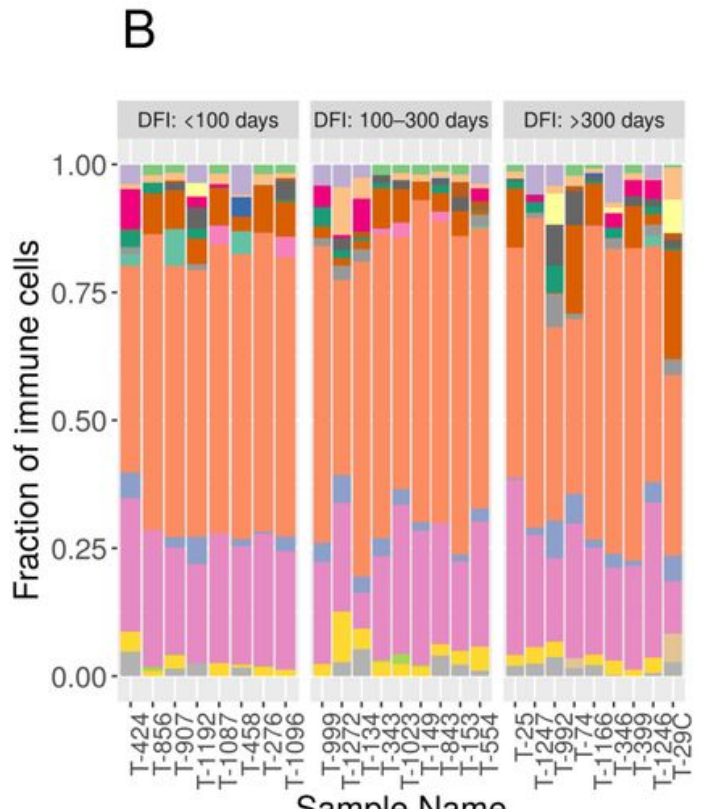

Sample Name
B cells naive

B cells memory

Plasma cells

T cells CD8

T cells CD4 naive

T cells CD4 memory resting

T cells CD 4 memory activated

$T$ cells follicular helper

$T$ cells regulatory (Tregs)

T cells gamma delta

NK cells resting

NK cells activated

Monocytes

Macrophages MO

Macrophages M1

Macrophages M2

Dendritic cells activated

Mast cells resting

Mast cells activated

Neutrophils

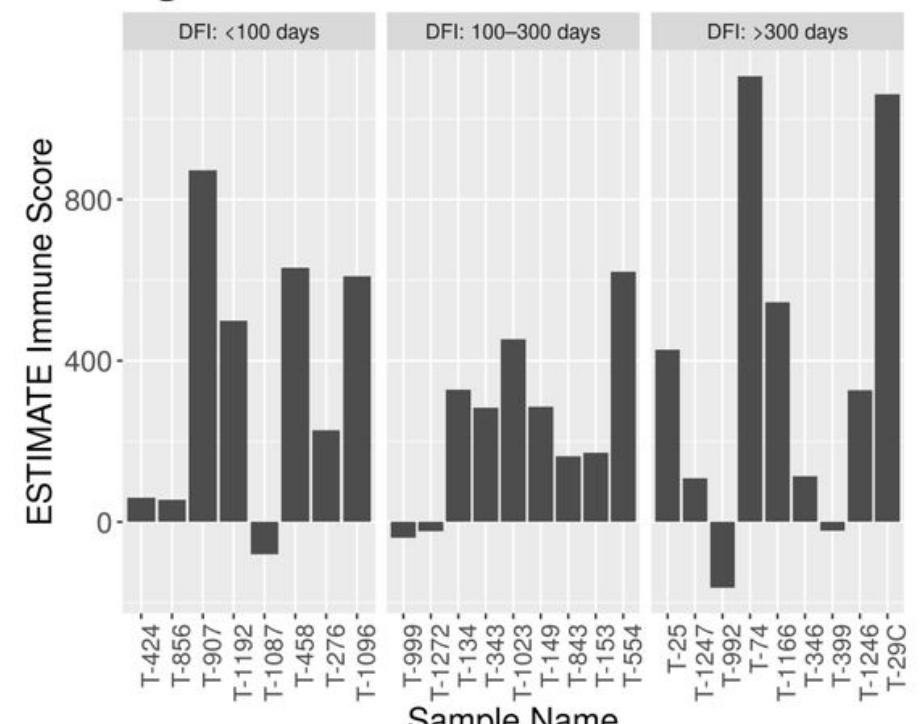

Sample Name

\section{Figure 5}

Representative figures of immunohistochemical staining of canine osteosarcoma samples. A-B. These samples were stained for CD3+ T-cell infiltration in A (low levels - T-1023) and B (high levels - T-1162). CD. These samples were stained with the MAC387 antibody against S100A9 to distinguish macrophage infiltration. C represents low levels (T-1162) and D represents high levels (T-856). E-F. Quantification of immunohistochemical labelling of T-cell (CD3+ cells) and Macrophages (MAC387 cells) across 19 and 22 samples, respectively. 


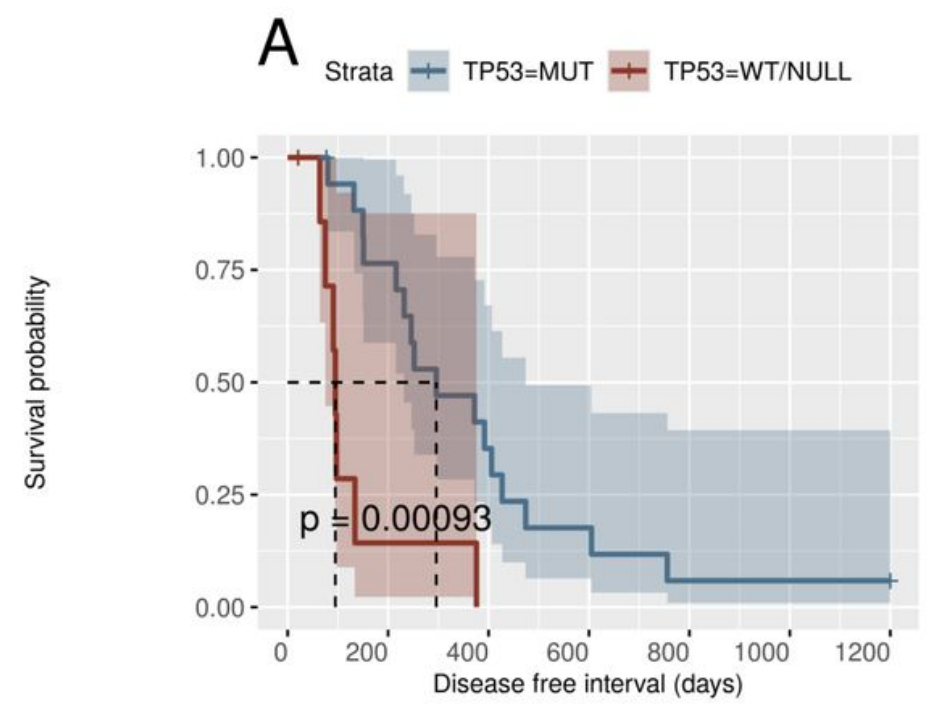

Number at risk

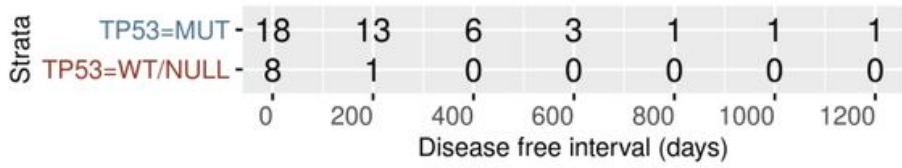

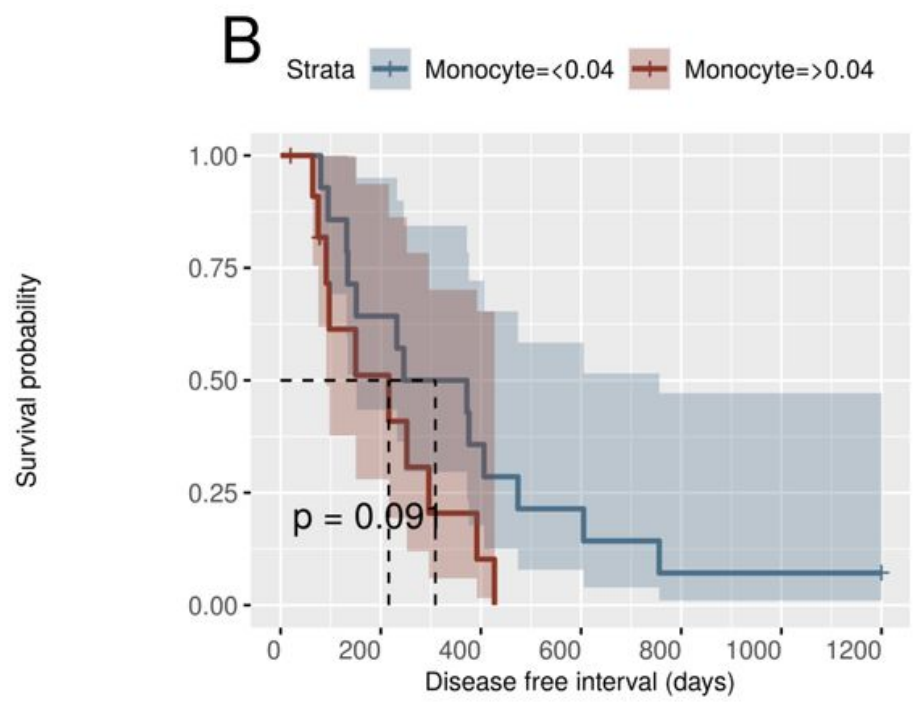

Number at risk

\begin{tabular}{|c|c|c|c|c|c|c|}
\hline$\underset{\widetilde{\sigma}}{\widetilde{\pi}}$ Monocyte $=<0.04-14$ & 9 & 5 & 3 & 1 & 1 & 1 \\
\hline ڤँ Monocyte $=>0.04-12$ & 5 & 1 & 0 & 0 & 0 & 0 \\
\hline $0^{\prime}$ & $200^{\prime}$ & $400^{\prime}$ & $\begin{array}{l}600^{\circ} \\
\text { free in }\end{array}$ & $\begin{array}{l}800^{\prime} \\
\mathrm{val}(\mathrm{d})\end{array}$ & s) & $1200^{\prime}$ \\
\hline
\end{tabular}

\section{Figure 6}

Profiling of immune cell infiltration using gene expression data. A. Pearson correlation matrix of immune cell expression score and immune staining levels of T-cell (IHC percent CD3+ cells) and macrophages (IHC percent MAC387 cells). We also correlated mutations per megabases (MB) and disease-free interval $(D F I)$ with other variables. Only the significant $(F D R<0.05)$ correlation values are plotted. $B$.

Quantification of 22 immune cell types using CIBERSORT. The samples are sorted by DFI. The most prevalent cell type in the tumor microenvironment were M0 and M2 macrophages. C. Immune score quantification using ESTIMATE R package.

\section{Supplementary Files}

This is a list of supplementary files associated with this preprint. Click to download.

- AdditionalFile1.xlsx

- AdditionalFile1.pdf

- AdditionalFile2.pdf

- nrreportingsummary.pdf 